\title{
Small Phase Space Structures and their Relevance to Pulsed Quantum Evolution: the Stepwise Ionization of the Excited Hydrogen Atom in a Microwave Pulse
}

\author{
Luca C. Perotti \\ Department of Physics, Texas Southern University, Houston, Texas 77004 USA
}

(Dated: November 21, 2018)

\begin{abstract}
Experiments have shown that the microwave ionization probability of a highly excited almost monodimensional hydrogen atom subjected to a microwave pulse sometimes grows in steps when the peak electric field of the pulse is increased. Classical pulsed simulations display the same steps, which have been traced to phase-space metamorphoses. Quantum numerical calculations again exhibit the same ionization steps. I show that the time-sequence of two level interactions, responsible for the observed steps in the quantum picture, is strictly related to the classical phase space structures generated by the above mentioned metamorphoses.
\end{abstract}

PACS numbers: 05.45.Mt, 03.65.-w, 32.80.Rm, 47.20.Ky

\section{INTRODUCTION}

In classical physics, integrability - even for nondissipative systems - is the exception [1]. Regular - both periodic and quasiperiodic - and chaotic classes of motion can coexist in phase space. Classes of motion can appear or disappear when the system parameters are changed; periodic orbit bifurcation points and thresholds for chaos are among the most widely known of the critical points where this happens.

The relevance of nonintegrable classical dynamics to quantum dynamics has been [2] and still is the source of much discussion, which has lately concentrated mainly on three problems: dynamical tunneling, both chaotic or resonance assisted [3], the construction of atomic nondispersive wave packets [4], and the ionization of Rydberg atoms [5].

Existing semiclassical theories predict a direct dependance of the quantum wavefunction on the corresponding classical physics, for instance via the classical action function (when it can be built). But the temporal regions of applicability of such theories generally are not well delineated, especially near the critical values and for chaos. Little is known about the case where a parameter is swept in time through a classical critical value. Under what conditions and to what extent can the evolution of a nonintegrable quantum system be "guided" by short-time nonadiabatic behavior present in its corresponding nonintegrable classical evolution?

One semiclassical dynamical system which has been for a long time the object of extensive experimental study is a highly excited hydrogen atom exposed to a partially ionizing short pulse of microwave electric field. If the atom is prepared in a static electric field collinear with the microwave field, and an extreme Stark state is excited, a one-dimensional approximation can be used, thus greately simplifying the treatment. Experimental and quantum numerical results are often found to be nearly classical, agreeing reasonably well with numerical predictions based on classical models. The classical electron dynamics is a microwave frequency sensitive mixture of regular motion, locally chaotic motion, and globally chaotic motion [6]. The last motion eventually results in ionization of the classical atom. Resonant regular motion is displayed as island features in stroboscopic surfaces of section of the phase space.

Let the initial principal quantum number of the atom be $n_{0}$. When the ratio $\omega_{0} \equiv n_{0}^{3} \omega$ of the microwave frequency $\omega$ to the initial Kepler orbit frequency $1 / n_{0}^{3}$ is near unity, resonant classical motion dominates the character of a number of semiclassical Floquet eigenstates which are experimentally populated at the peak of the pulse [6]. This inhibition of chaos produces an experimentally observed increase in the threshold field for ionization in the quantum system [7]. Bounding the island region in phase space is a locally chaotic region which can be crossed by the system during the rise and fall of the pulse [8]. Such crossings are examples of a phase-space transport process which would not occur in a fixed-field experiment $[9,10]$. They provide an explanation for observed nearly classical final quantum state distributions which are double peaked as a function of the principal quantum number [11]. I shall call principal primary resonance zone the $\omega_{0}=1$ resonance island together with the surrounding band of local chaos.

For different constant values of the microwave field strength, the structure of the resonance zones can be different. The structural changes have been called phase-space metamorphoses [12], and it has been suggested that they could influence the microwave ionization process. For example, in Ref. [12] it was shown that the destruction of the last invariant curve between a primary resonant zone and the global chaos above it can lengthen the classical electron's escape time for ensembles of initial conditions in the global chaos region. Here I am interested in the metamorphoses of the internal structure of the resonance islands themselves. These metamorphoses are associated with periodic orbit bifurcations which generate, within the primary resonance zones, new "secondary" resonance Kolmogorov-ArnoldMoser (KAM) island chains, and with the destruction of these same secondary resonance island chains.

Laboratory observations have been reported of sequences of steps in the (experimentally defined) ionization of 
almost monodimensional Hydrogen Rydberg atoms versus the peak field strength of the microwave pulse [13]. Classical numerical simulations reproduce such steps. These classical step have been shown to be due to metamorphosis-induced variations of the probability for the transport in phase space responsible for ionization [13]. Together with more extensive numerical evidence and a more detailed analysis of the classical process [16], I now present an explanation of the good agreement of the quantum simulations with the classical ones: even if, in terms of Floquet states, the quantum evolution is dominated by a sequence of two level processes (avoided crossings traversed by the system) and can therefore be viewed as deeply quantum, the interactions between these couples of states exist because of the classical secondary island chains.

The present paper is organized as follows: in section II I present the system and the numerical techniques I used to investigate it; the results of my investigation are presented in section III. Section IV is dedicated to the interpretation of these results; section $\mathrm{V}$ finally sums up my findings.

\section{THE SYSTEM AND NUMERICAL METHODS}

The system I investigate is a $1 D$ model for a stretched highly excited hydrogen atom in collinear static and monochromatic microwave electric fields [14]. In atomic units the Hamiltonian reads

$$
H=\frac{p^{2}}{2}-\frac{1}{z}+z\left[F(t) \sin \left(\omega t+\phi_{0}\right)-F_{s}\right], \quad z \geq 0
$$

where $F(t)$ is the strength of the microwave field, $\phi_{0}$ is its initial phase, and $F_{s}$ is the static field strength. To simulate the experimental situation described in Ref. [13], the envelope of the microwave pulse is chosen to be

$$
F(t)=F^{\max } \sin \left(\pi t / T_{p}\right)
$$

where $T_{p}=7.78 n s$ is the length of the microwave pulse. For most of the simulations in the present paper this corresponds to 136 microwave periods.

As is costumary for this system I shall use classically scaled parameters [14]: $F_{0}^{\max }=n_{0}^{4} F^{\max }, F_{s 0}=n_{0}^{4} F_{s}$, and $\omega_{0}=n_{0}^{3} \omega$. The scaled frequency is furthermore corrected to the first order in $F_{s 0}$ to compensate for the Stark shift of the atomic frequencies [6]:

$$
\omega_{0}^{\prime}=\omega_{0} /\left(1-3 F_{s 0}\right)
$$

\section{A. Classical methods}

Following Ref. [15], classical numerical integration in Ref. [16] was performed in free-atom action angle variables $(I, \theta)$, valid when the electron's energy is negative and defined by the equations:

$$
\begin{gathered}
z=2 I^{2} \sin ^{2}(\xi / 2), \\
p=(1 / I) \cot (\xi / 2),
\end{gathered}
$$

where the eccentric anomaly angle $\xi(\theta)$ is defined by $\theta \equiv \xi-\sin \xi$.

To avoid equations of motion containing terms which diverge as $z$ approaches zero, a dummy time $\eta$, defined by $d t \equiv(1-\cos \xi) d \eta$ was also introduced [17]. The true time t increases monotonically with $\eta$. Newton's equations of motion then become

$$
\begin{aligned}
& d I / d \eta=-I^{2} \sin \xi\left[F(t) \sin \left(\omega t+\phi_{0}\right)-F_{s}\right] \\
& d \xi / d \eta=1 / I^{3}+2 I(1-\cos \xi)\left[F(t) \sin \left(\omega t+\phi_{0}\right)-F_{s}\right] \\
& d t / d \eta=1-\cos \xi
\end{aligned}
$$

The above three ordinary differential equations were numerically integrated using a fixed step, fourth order RungeKutta routine. The ensemble of initial conditions $\left(\theta_{0}, I_{0}\right)$ was chosen so that, to first order in $F_{s}$, the (classical) electron energy $E(t=0)$ be equal to the value of the energy of the experimentally prepared initial quantum energy eigenstate:

$$
-\frac{1}{2 n_{0}^{2}}-\frac{3}{2} F_{s} n_{0}^{2}=E(0)=-\frac{1}{2 I_{0}^{2}}-F_{s} I_{0}^{2}\left(1-\cos \xi_{0}\right)
$$


Given a value of $\theta_{0}\left(\xi_{0}\right)$, this equation determines a corresponding value of $I_{0}$.

Classical values for the "ionization" probability $P_{I}$ at the end of the pulse were averaged over uniform distributions of the initial angle $\theta_{0}$ and microwave field phase $\phi_{0}$. The "ionization" probability contains two contributions. One comes from trajectories which are terminated at some time during the pulse where the instantaneous value of the energy $E$ exceeds the value $-2 \sqrt{F_{s}}$ for rapid ionization in the static field alone. To match the experimental definition of "ionization" [13], the other contribution comes from trajectories whose final value of $E$ corresponds, according to equation (2) above, to energy eigenstates with quantum numbers outside the interval $n \in[50,90]$.

Stroboscopic surfaces of section of the long-time evolution in $(\theta, I)$ space were also computed for $F(t)$ equal to various constants $F[16]$. These reveal the long-time structures existing in phase space for the various values of $F$ instantaneously traversed during the microwave pulse. For a constant amplitude microwave field, the action-angle generalized phase space (in the three coordinates $I, \theta$, and $t$ ) is a well known [6] admixture of zones of regular and chaotic motion. Zones of regular electron motion include those occupied by nested vortex tubes and those of the field-modulated atom [8]. Chaotic zones include zones of local chaos and of global chaos. Stroboscopic surfaces of section (Poincaré maps) reflect these zones in the full phase space as characteristic zones in the action-angle $(I, \theta)$ subspaces ("sectioned" phase space): the vortex tubes produce resonance island zones and the local chaos produces zones of irregular motion which have been called "separatrix" zones [18] (the actual separatrix of integrable systems is "broken" in the nonintegrable driven hydrogen atom problem and becomes a chaotic layer which grows with increasing microwave field strength). These structures are self-similar: secondary resonance zones are found within primary resonance zones and so on; but while all primary resonance zones are born at zero microwave field, periodic orbit bifurcations produce secondary resonance zones at nonzero field values.

The comparison of instantaneous ensemble distributions in phase space with these long time surfaces of section, for various times $t$ during the pulse [13] has proven a useful tool for the understanding of the evolution of the ensemble itself: when a phase space structure influences the pulsed evolution, the ensemble appears deformed by it in a characteristic way. For example, an ensemble of points initially on a straight line is stretched by a resonance island into a whorl [19] as the points of the ensemble move along the island's invariant curves. Local whorl-like deformations of the ensamble are therefore indication of the influence of an underlying island which can be identified with the help of the surface of section at that time. Similarly, local hyperbolic chaos deforms the ensemble into tendrils [19].

\section{B. Quantum methods}

The time evolution of the quantum system is evaluated by numerical integration of the Schrodinger equation with $H$ given by eq.(1) on a finite subset of the (bound) free atom basis $\psi_{n}(z)$ chosen big enough so that the probability reflected by the borders is small [15]. Given an initial state $\psi(z, 0)=\Sigma_{n} C_{n}(0) \psi_{n}(z)$, the equation for the evolution of the expansion coefficients $C_{n}(t)$ is

$$
\begin{array}{r}
i \frac{d C_{n}(t)}{d t}=E_{n} C_{n}(t)+\mathcal{F}(t) \Sigma_{m} Z_{n, m} C_{m}(t), \\
\mathcal{F}(t)=\left(F(t) \sin \left(\omega t+\phi_{0}\right)-F s\right),
\end{array}
$$

where $Z_{n, m}$ is the matrix element of the operator $z$ between the states $n$ and $m$. We approximate $\mathcal{F}(t)$ with the function $\mathcal{F}^{\prime}(t)=\mathcal{F}(t) \Delta t \Sigma_{k} \delta(t-k \Delta t)$ which tends to $\mathcal{F}(t)$ for $\Delta t \rightarrow 0$ (in the sense that the integral of their difference over an arbitrary time interval goes to zero as $\Delta t$ ) [15]. This procedure involves unphysical parameters which have to be carefully chosen as not to falsify the results of the integration: I discuss my choices in Appendix A.

The initial state for our simulations is taken to be the eigenstate with quantum number $n_{0}$ of the atomic Hamiltonian in the static field alone, calculated in the chosen finite subset of the free atom basis.

\section{Quasienergy curves}

Like in the classical case, an understanding of the dynamics at constant microwave amplitude will help illuminating the pulsed dynamics. If $F(t)$ is a constant, Schrodinger's equation is a differential equation with time-periodic coefficients. Floquet's theory is therefore applicable [20] and tells us that the equation has solutions in the form

$$
\psi_{i}(t)=\Phi_{i}(t) e^{-i \varepsilon_{i} t / \hbar}, \quad \Phi_{i}(t+T)=\Phi_{i}(t)
$$

where $T$ is the period of the microwave, the constants $\varepsilon_{i}$ take the name of quasienergies and the functions $\psi_{i}(t)$ are called quasienergy (or Floquet) states. For periodic systems quasienergies take the place of energies in the description 
of the dynamical properties of the system. It can be easily seen that the same physical state $\psi_{i}(t)$ can be written as

$$
\psi_{i}(t)=\left[\Phi_{i}(t) e^{-i 2 \pi n t / T}\right] e^{-i \varepsilon_{i} t / \hbar+i 2 \pi n t / T}
$$

where $\Phi_{i}(t) e^{-i 2 \pi n t / T}$ is again a T-periodic function. We therefore have an infinity of replicas of the quasienergy spectrum shifted by $2 \pi \hbar / T$ one respect to another. It will then be sufficient to restrict ourselves to the energy interval $[0,2 \pi \hbar / T)$ (first Brillouin zone) to have all the levels. In particular, since the free atom has a continuum, this too will be brought into the first Brillouin zone. Due to the presence of the free atom continuum, the microwave transforms all the free atom states into resonances with finite widths $\Gamma_{i}$ : the quasienergy spectrum for an atom in microwaves is therefore absolutely continuous (no point spectrum exists) [21].

For the actual calculation of the quasienergies it is useful to resort to the time evolution operator $G$ over one period $T$ of the microwave (Floquet operator). There isn't just one Floquet operator, but a whole family of them, parametrized by the phase $\phi=\left(\omega t_{0}+\phi_{0}\right)$ of the field at the beginning of the period. While the eigenfunctions $\Phi_{i}(\phi)$ of each member of the family are different and represent the different spatial structures of the states at different times during the microwave period, the family shares the same eigenvalues $G_{i}$. The eigenvalues $G_{i}$ of the Floquet operator and the quasienergies are connected by the relationship $G_{i}=e^{-i \varepsilon_{i} T / \hbar}$. One common procedure to obtain the quasienergies is therefore to numerically calculate a one period evolution operator and diagonalize it thus obtaining the $G_{i}$ s. The above relationship then gives us the quasienergies on the circle $[0,2 \pi \hbar / T)$.

To investigate the evolution of a pulsed system, and also -as we shall see in Appendix A- to control numerical errors and approximations, it is useful to plot the quasienergies as functions of the microwave field strength $F$. The resulting quasienergy curves (see e.g. Fig. 1) help us connect the characteristics of the system at different values of the microwave field strength. The quasienergies change with $F$ and undergo a sequence of avoided crossings of different widths. Since in the $1 D$ hydrogen atom all the quasienergies fall into the same symmetry class [22], the Von Neumann-Wigner theorem [23] tells us that they all repel each other; this implies the absence of real level crossings. Just like in the simulations of the pulsed system, the Floquet operator is calculated on a truncated basis and some caution has to be exerted to avoid spurious results. A study of the quasienergy curves themselves can help us in deciding what parts of what curves to trust [24]: see Appendix A.

\section{Quantum nonlinear resonances}

The behaviour of a grouping of levels strongly interacting (repelling each other) at or close to zero microwave field is particularly evident in Fig. 1. I have marked them as darker lines. If we look at the (zero microwave field) energies of these levels we see that each level is close to a quantum resonance

$$
\Delta E \equiv E_{n+r}-E_{n}=s \omega \hbar
$$

with any of the other levels of the same grouping; the ratio $s / r$ being equal to 1 for any two levels of the grouping. This grouping represents on the $(E, F)$ plane what Ref. [25] calls a nonlinear quantum resonance: a finite (because of level anharmonicity) number of quantum states whose interaction is due to the existence of a (primary) classical resonance zone, in this case the $\omega_{0}^{\prime}=1$ resonance zone. The structure these levels form can be described as an excitation of the system induced by the perturbation: Ref. [26] calls it a "Floton". The state of the Floton is decribed by the new quantum number $k$ given in Fig. 1 next to the free atom one $n$; a simple interpretation of it in terms of pendulum approximation is given in Appendix B 2. The most stable state is the ground one $(k=0)$; the excited states $k=1,2, \ldots$ are progressively shorter lived [27], as long as we consider only the properly resonant states: those for which the inflection point of the corresponding quasienergy curve in Fig. 1 lies to the left of the considered value of the microwave field strength $F$ (see Appendix B 2).

\section{Husimi function}

One of the most widely used representations of a wavefunction in phase space is the Husimi function [28]. Given a quantum function $\psi(t)$, its Husimi function at a point $\{\langle p\rangle,\langle q\rangle\}$ in phase space is the projection (in atomic units) $\rho_{H}(\langle p\rangle,\langle q\rangle, t)=\left|\left\langle\phi_{\langle p\rangle\langle q\rangle} \mid \psi(t)\right\rangle\right|^{2}$ of $\psi(t)$ on a (minimum uncertainty) packet $\phi_{\langle p\rangle\langle q\rangle}$ centered on that same point in phase space and called the "coarse graining function". In action-angle space, due to the periodicity in the angle variable, the standard (position-momentum space) choice of coarse graining function [28] is not possible. Following Ref. [29] I therefore take

$$
\phi_{\langle I\rangle\langle\theta\rangle}=\Sigma_{n=0}^{\infty}\left[\frac{\alpha^{(\alpha n+1)}}{2 \pi \Gamma(\alpha n+1)}\langle I\rangle^{\alpha n} e^{-\alpha\langle I\rangle}\right]^{1 / 2} e^{i 2 \pi\langle\theta\rangle n} \psi_{n} .
$$


Tests with different values of the width parameter $\alpha$ have given - both for Ref. [29] and for me - Husimi functions having essentially the same shape when $\alpha<n_{0}$. For $n_{0} \gg 1$ the classical zones in phase space are reflected in the character of the Floquet eigenstates (FE), especially as seen through their Husimi functions (see refs. [24, 29] and - for semiclassical FE - Ref. [8]). Thus regular FE can be either resonance island FE or modulated atom FE. Irregular FE include the separatrix FE, possibly "scarred" by a high electron probability density in phase space along an unstable periodic orbit $[12,27]$; in sectioned phase space this corresponds to a maximum of the Husimi function on the unstable fixed point which (surprisingly) has a simple interpretation in pendulum approximation [26]. At intermediate values of microwave field strength, these scarred separatrix FE exhibit partial quantum localization along the unstable periodic orbits (rather than full localization on nested vortex tubes), and otherwise are distributed within a bounded region of phase space classically occupied by chaotic trajectories $[8,27]$. The comparison of Husimi functions for the quasienergy states with the classical surfaces of section at the same values of $\omega, F$ and $\phi_{0}$ has often proven quite instructive. Comparison of Husimi functions and classical instantaneous ensembles for the pulsed simulations is also possible, but I found them less useful in the present study.

\section{RESULTS}

In Ref. [13], the large secondary resonance island chains encircling the stable fixed point of the Poincaré map at the center of the $\omega_{0}^{\prime}=1$ resonance zone were identified as the cause of the sequence of steps in the classical ionization probability vs. peak microwave field $F_{0}^{\max }$ graphs, of which three examples are shown as full lines in Fig. 2. These chains are born from the distruction of those periodic orbits of the Poincaré map laying within the $\omega_{0}^{\prime}=1$ primary resonance zone which have periods whose ratio to the microwave period equals the ratio $q / p$ of two (relatively prime) integers [13]. As $F_{0}$ is increased, The most visible chains have $p=1$ ("integer resonances") and the number $q$ of individual islands in them decreases with $F_{0}$ [13]. The size of an island chain grows rapidly with $F_{0}$ above a bifurcation point $F_{0 q / p}$ in qualitative agreement with the simple pendulum approximation model described in appendix B 1.

The arrows in Fig. 2 show the field strength values at which the $p=1$ island chains destabilize (become completely chaotic) and are destroyed, for $q=3$ to 6 . Each of these values is very close to a step onset, strongly suggesting a connection between the ionization steps and the secondary island chains; the connection is confermed by the evolution in phase space of the classical trajectory ensemble. Four examples - of the many I have calculated in Ref. [16]- are given in Figs. 3-6: in all cases we are at the base of an ionization step and a sizable part of the ensemble (marked by bigger dots) finds itself at the peak of the pulse inside the islands of some secondary chain: $q=6$ for Fig. $3, q=5$ for Fig. $4, q=4$ for Fig. 5 and $q=3$ for Fig. 6 . Together with the big whorl of the points trapped within the primary island, the marked points make up most of the sub-ensemble surviving the microwave pulse, as can be seen from the last snapshot of each sequence. Whorls and tendrils developed by the ensemble at intermediate times during the rise of the pulse indicate the influence of other secondary chains with higher $q$ 's. The relevant chains were in each case identified by comparing the snapshot of the ensemble with the constant field stroboscopic surface of section taken at the same value of $F_{0}$; examples are shown in Figs. 7-10. These comparisons reveal the following classical time sequence of events responsible for a step in Figure 2 (a more schematic description was given in Ref. [13]). The ensemble initially is located on a nonresonant phase-space KAM curve which spans the full range $[0,2 \pi]$ of the angle variable. The pulse peak field $F_{0}^{\max }$ must of course be high enough that by the time we are at the peak of the pulse the locally chaotic region of the principal primary resonance has merged with the global chaos above (the last KAM curve separating them has broken) so that classical ionization is possible.

A) For $F_{0}(t)$ near 0.01 , the ensemble almost entirely crosses the locally chaotic region to enter the $\omega_{0}^{\prime}=1$ island region; the wide "nose" the ensemble develops at the crossing (see the second snapshots in Figures 3-6) tells us that after the crossing the spread in resonance action $J$ (action of the slow motion around the stable fixed point of the Poincaré map) is quite wide.

B) After the crossing there is a time interval of slow near adiabatic ensemble evolution, having an approximate frequency given by eq. (B6). The ensemble is at the same time partially "adiabatically squeezed" in the angle variable [30] toward the center of the island (fourth to sixth snapshots in Figures 3 and 4, third to fifth snapshots in Figure 5 and third to fourth snapshots in Figure 6). If $\left|\omega_{0}^{\prime}-1\right|<0.05$, much of the ensemble approaches and stays close to the center of the island.

C) A bifurcation sequence with decreasing $q$ has been occurring with increasing $F_{0}(t)$. For each $q$ the size of the corresponding island chain grows rapidly [13]. Within a field strength increment between $5 \%$ and $20 \%$, this size reaches a value about equal to the nominal size of the entire primary island region $\left(\Delta n=8\right.$ to 11 depending on $\left.F_{0}(t)\right)$. The size of the individual islands of the chain grows too; for a given size $\Delta I$ of the chain the size of its islands appears to increase with decreasing $q$.

D) A study of surfaces of section at $F_{0} \lesssim 0.01$ (crossing field strength) shows that the secondary island chains with $q \gtrsim 8$ are all born before the crossing; most of them (those with $q \gtrsim 12$ ) have moreover already grown to full size and 
destabilized. The remaining secondary island chains can and - for $q<8$ - do influence the evolution of the ensemble as can be seen in the superpositions of ensembles over instantaneous surfaces of section shown in Figs. 7-10: parts of the ensemble are "trapped" by these chains. By "trapped" I mean that once the points of a part of the ensemble are inside the islands of a chain, they remain inside those islands and follow the chain in its motion (during the rise of the pulse) toward the periphery of the primary resonance zone [31]. Note that the direction of this motion is the opposite of that of the adiabatic "squeezing" we observed in step B. This inversion of motion of parts of the ensemble within the primary resonance island is a clear indication of their trapping by the secondary resonance zone. Finally, since the span, in the resonance action $J$, of the islands of the secondary chain is usually not big, it is important that a sizable portion of the ensemble be at some time within the same small range of $J$ so as to be able to be trapped by the islands of the secondary chain. This is made possible by the "squeezing" we have seen at point B.

For each of the four cases in Figs. 3-6 I have marked with darker dots that part of the ensemble which at the peak of the pulse is within the relevant island chain: the motion of the "trapped" part of the ensemble toward the periphery of the primary resonance zone is particularly clear in Figures 5 and 6 . It is also evident that different island chains act on different parts of the ensemble: the higher is the $q$ of a chain the further from the center of the primary resonance zone is the part of the ensemble it "traps". Parts of the ensemble closer to the center of the resonance zone do not "see" the chain when it grows through them: they are traversed diabatically (motion within the secondary islands is too slow to be "seen" by the points of the ensemble for the chosen switch-on time). Only at a certain distance from the center of the primary resonance zone a given island chain becomes "visible" to the ensemble, and this leads us to believe that, together with the size of the chain and the size of the islands of the chain, also the (average) frequency of motion within those islands must increase with $F_{0}$ (just like the frequency of motion within the primary island: see eq. (B5)). Moreover, for a given pulse length, the distance from the center at which this happens increases with the $q$ of the chain, suggesting that, for any given distance from the center, the higher is $q$ the lower is the frequency of motion on the islands of the chain.

Now that we have part of the ensemble trapped in the secondary chain, we have two possibilities : $\mathrm{E}$ and E' $+\mathrm{F}$ '.

E) As long as $F_{0}^{\max }$ remains smaller than the one necessary to destroy the islands of the secondary chain, the only part of the ensemble which can and does ionize is the one in the chaotic region of the primary resonance zone; no further ionization is possible. We are on the plateau of the step.

E') If we instead further increase $F_{0}^{\max }$, the island chain becomes locally chaotic, and then "globally" chaotic (that is: it merges with the chaotic band surrounding the primary resonance island and finally with the global chaos above), all within a further field strength increment of about $5 \%$ : rather abruptly a sizable part of the phase space becomes globally chaotic. It is this sudden increase of the globally chaotic region that causes the step in ionization probability. The growth of the band of local chaos is reflected in the changed appearance of the parts of the ensemble trapped in the chain: whorls, connected to motion within the stability islands, are substituted by tendrils, connected to motion in the chaotic band. the last two snapshots in Fig. 7 show such a transition for the $q=6$ island chain; the association of tendrils with the destruction of chains is also clear in the second snapshot in Fig. 9 (again the chain is the $q=6$ one) and in the third $(q=5)$, fourth $(q=4)$ and sixth $(q=3)$ snapshot in Fig. 10.

F') The chaotic trajectory subensemble released by the island chain destruction event E' produces ionization during a time interval of about $30 \%$ of the pulse length, within the relatively constant central part of the half sinewave pulse.

The examples I have given in Figures 3-6 and 7-10 make clear that "trapping" in the secondary island chains is possible for the experimental pulse times of about 131 microwave periods. Longer pulse times mean slower growth of $F_{0}(t)$ resulting in even more effective trapping, as can be seen from Fig. 14 in Ref. [32]: the sharpness of the ionization probability steps improves with increasing pulse times.

An important implication of the above scenario is that, for fixed values of the static field $F_{r}^{s}$ (rescaled at the resonant action $I_{r}$ defined by $\left.\omega I_{r}^{3} /\left(1-3 F_{s} I_{r}^{4}\right)=1\right)$, the position in $F_{r}^{\max }$ of the steps depends only on the destabilization of the island chain and not on the initial conditions of the ensemble. The initial action (and the pulse time) on the other hand determine the heights of the steps. As an example I have plotted classical ionization probability data for rescaled frequencies $\omega_{0}^{\prime}$ between 0.9325 and 1.0666 first as a function of $F_{0}^{\max }$ (Fig. 11.a) and then as a function of $F_{r}^{\max }$ (Fig. 11.b): notwithstanding the great difference in height, the position of the steps in Fig. 11.b is approximately the same $\left(F_{r}^{s}\right.$ is slightly different from curve to curve); while this is clearly not the case in Fig. 11.a [33].

Parallel quantum numerical simulations are shown as dashed curves in Fig. 2. In Figure 2.a. The classical steps labeled $q=3-6$ are reproduced quite well. They appear even sharper in the quantum simulations than they are in the classical ones. On the other hand the oscillations of the plateau just before the $q=3$ step are much more pronounced than the classical ones. The absence of a $q=2$ quantum step in the figure most likely reflects the fact that the $q=2$ island chain in (sectioned) phase space is not large enough to significantly support a Floquet eigenstate (even if their height is comparable to that of the islands of other chains, the two islands are extremely narrow: never more than about 0.1 radians in the angle variable). Figure 2.b shows another case where the above comments again apply: the quantum curve follows quite closely the classical one up to the $q=3$ step but then directly rises to $100 \%$ ionization probability, skipping the $q=2$ step. Figure 2.c finally shows the quantum and classical steps for a third 
case; this time $\left|\omega_{0}^{\prime}-1\right|=0.073$ is big enough that the ensemble does not penetrate all the way to the island's center. I believe this to be the cause of the absence of the $q=2$ step in the classical curve: by the time the $q=2$ island chain is born, the primary island is so small that all of the ensemble is outside of it in the chaotic region and cannot be trapped.

The good agreement between classical and quantum simulations is not limited to the final ionization probability; the evolutions during the pulse are remarkably similar too. In Fig. 12 I show a comparison of the quantum and classical ionization probability versus time: the shape of the curves is always very similar even if the final ionization probability is often not exactly the same.

\section{Classical and quantum resonances}

To explain this agreement between classical and quantum ionization results, we now explore the connection between higher order classical resonances and quantum resonances at nonzero microwave field (avoided crossings in the quasienergy plots). We start with some general considerations which will then allow us to locate the avoided crossings related to the secondary resonance zones within the principal primary resonance island [16].

For simplicity, let us start considering an isolated avoided crossing of two energy levels $E\left(n_{i}, F\right), i=1,2$ with $F$ some perturbation parameter. We assume that we have been able to separate the complete Hamiltonian $H$ of the system in two parts, $H_{0}$ and $H_{1}$, such that only the interaction responsible for the separation of the levels at the crossing is included in $H_{1}$ and the levels $E_{0}\left(n_{i}, F\right)$, eigenvalues of $H_{0}$, actually cross at the field $F_{c}$ of closest approach of the levels $E\left(n_{i}, F\right)$ of $H$. We moreover assume that $E_{0}\left(n_{i}, F\right)$ is the quantized version of a classical function $E_{0}(I, F)$ which is smooth for $I \in\left[n_{1}, n_{2}\right]$. Since the classical phase space can be divided into regions corresponding to different kinds of motion and discontinuities in action occur at the borders of these regions [34], the above condition requires us to assume that for all relevant $F$ 's the two levels of $H_{0}$ we are considering correspond to the same kind of classical motion (their support is in the same region of the classical phase space of $H_{0}$ ). When these conditions are verified, at the field strength value $F_{c}$ where the levels $E_{0}\left(n_{i}, F\right)$ cross the average slope of the function $E_{0}\left(I, F_{c}\right)$ for $I \in\left[n_{1}, n_{2}\right]$ is 0 . There must therefore exist an action $I_{r} \in\left[n_{1}, n_{2}\right]$ verifying the classical resonance condition $\partial E_{0}\left(I, F_{c}\right) /\left.\partial I\right|_{I_{r}}=0$ (Rolle's theorem). For such an avoided crossing and for small values of the perturbation, a relationship has been derived in Ref. [35] expressing the splitting at the avoided crossing as a function of the area of the related resonance zone, both functions of the interaction Hamiltonian $H_{1}$.

If the perturbation Hamiltonian $H_{1}$ is time dependent and periodic with frequency $\omega$, then the avoided crossing is between quasienergy states, the quantum resonance condition for the (no more crossing) energy states of $H_{0}$ is eq. (4) and the classical resonance condition is $\partial E_{0}\left(I, F_{c}\right) /\left.\partial I\right|_{I_{r}}=s \omega / r$.

Let us now take $H_{0}$ to be the "pendulum approximation" Hamiltonian

$$
H^{(1)}=\left[-\frac{1}{2 I^{2}}-\frac{3}{2} F_{s} I^{2}-\omega I\right]+0.325 F I_{r}^{2} \cos \varphi
$$

which describes the resonant motion within the principal primary resonance zone $s / r=1$ (see appendix B 2 ). Its numerically calculated levels for the parameter values of Fig. 2.a are shown in Fig. 13 (again see appendix B 2 for details). After each level's inflection point (which is when the level gets "trapped" [25] into the region of libration motion), the functional dependence of the energy of the eigenstates of $H^{(1)}$ on the pendulum quantum number $k$ is that of the classical energy on the action $J$ within the (primary) "resonance" zone of libration motion (see fig 23 in appendix B 2). Since this dependence is smooth we can apply the argument above: crossings between states with $\Delta k=q$ and energy distance $\Delta E=p \omega$ are related to the $\Omega=\omega p / q$ classical secondary resonance condition where $\Omega$ is the "slow motion frequency" $|\partial H / \partial J|$. In Fig. 13 I have marked the crossings of the pendulum approximation levels with different symbols for the different $p / q$ ratios (connected by lines to guide the eye); arrows mark the classical bifurcation fields eq. (B7) for the same $p / q$ ratios. As expected from the argument above, all the crossings with a given $p / q$ ratio lay to the right of the (pendulum approximation) bifurcation field for the secondary resonance zone with the same $p / q$ ratio. Also, the crossings with the same $p / q$ ratio are not all at the same field but the higher the average $k$ of the levels crossing the higher the field at which the crossing takes place, in agreement with the moving (with increasing $F$ ) of the center of the classical secondary resonance zone toward higher pendulum actions [16].

We can now turn to the quasienergy levels of the full problem, of which the pendulum ones are a good approximation near the $s / r=1$ quantum nonlinear resonance at low microwave field strengths [36]. The true levels' avoided crossings corresponding to the approximate levels' crossings, even if shifted (at high microwave field strengths) with respect to these latter ones, are related to the same secondary resonance zones and therefore lay to the right of the numerical classical bifurcation fields for the same $p / q$ ratios, as can be seen in Fig. 1 (where we use the same conventions as in Fig. 13) [16]. 
The identification of the crossings related to these secondary island chains is supported by the Husimi functions I calculated for the Floquet eigenstates undergoing the avoided crossings: some of them are shown in Figs. 1418. Before discussing them let us first introduce one last concept: that of crossing region. For $F \ll F_{c}$ the two quantum states $E\left(n_{i}, F\right)$ are practically noninteracting and coincide with $E_{0}\left(n_{i}, F\right)$; approaching $F_{c}$ they begin to interact more. This interaction is reflected in the mixing of the two states which is maximum at $F=F_{c}$ where the two eigenstates of $H$ undergoing the avoided crossing are linear combinations in equal parts of the two (crossing) eigenstates of $H_{0}$. After that, the mixing decreases until, for $F \gg F_{c}$, they are again the two pure $E_{0}\left(n_{i}, F\right)$ states we started with but now the state $n_{1}$ has the spatial structure $n_{2}$ had before the crossing and viceversa, as if the two levels had switched. The quantum resonance itself as defined by eq. (4) is therefore only the central point of a region in $F$ where the two levels interact with each other. The values of $F$ such that the separation of the noninteracting levels $E_{0}\left(n_{i}, F\right)$ equals the interaction term can be taken as approximate boundaries in $F$ of the region where there is significant mixing [24]; we call this region the crossing region. Away from crossing regions the Husimi functions of the primary resonance eigenstates appear approximately as circular ridges, apart from the $k=0$ one which is approximately a Gaussian peak [24]. All these ridges have their center in the center of the classical principal primary island and the higher the resonance quantum number $k$, the higher their average radius. Examples are the Husimi functions before and after the avoided crossings shown in Figures 14-18. At a crossing related to classical secondary chain of $q$ islands, the exchange of the spatial structures of the two eigenstates involved happens in a characteristic way: the outer ridge moves inwards avoiding ("seeping" around) the $q$ regions of the islands of the chain while the inner ridge moves outwards right through these regions, avoiding the regions around the unstable periodic orbit of the island chain and sometimes developing $q$ peaks corresponding to the islands of the secondary chain. Often this $q$-fold structure of the states is not equally visible for both states at the same value of the microwave field strength $F$ : for most of the cases studied the structure is most visible for the outer states at slightly lower values of $F$ than for the inner state. Fig. 14 is a particularly clear case: the Husimi functions of the $k_{1}=2$ and $k_{2}=7$ resonance states both develop at their crossing the expected fivefold structure. Figure 15 shows instead the $(q=7)$ crossing of the $k_{1}=3$ and $k_{2}=9$ states: since the $k_{2}=9$ state is here almost a separatrix state (its Husimi function has not yet taken the typical resonance state shape and is peaked around the unstable fixed point of the Poincaré map [27]) we are nominally outside the range of applicability of my semiclassical argument, still the sevenfold structure is visible. In other cases the process can be complicated by the interaction with other states but the $q$-fold structure is always present. Figure 16 shows the $(q=6)$ crossing of the states $k_{1}=2$ and $k_{2}=8$ where the lower "adiabatic" state (with respect to the $q=6$ crossing) is made up of the two interacting states B and C. In Fig. $17\left(q=5, k_{1}=0, k_{2}=5\right)$ it is the upper "adiabatic" state which instead is made up of the two states A and C. Finally Fig. 18 shows a case $\left(q=4, k_{1}=0, k_{2}=4\right)$ where the three level interaction results in the adiabatic state "B" assuming before the crossing the role of upper "adiabatic" state and after the crossing the role of lower "adiabatic" state, thus maintaining the same resonance quantization $k_{2}=4$. These Husimi functions make clear that at the crossing regions I have marked in Fig. 1 the crossing eigenfunctions do see (and reflect in their structure) the $q$ dips of the classical dynamical potential [37] evidentiated in the surfaces of section by the $q$ islands of the secondary chain they are related to.

We now have to show that these crossings are in effect those where most of the population transfer causing the ionization steps happens during the microwave pulse. To do this we plot the projections of the wavefunction on the instantaneous Floquet eigenstates at each period of the microwave during the pulse. For the four values of the peak microwave field strengths of Figs. 3-6 these projections are shown in Figs. 19-22. They give us a dramatic representation of the importance of the avoided crossings we have individuated (marked here with the same symbols as in Fig. 1). After the initial spread of the population over the states $k=0-3$, the population is further spread out to high $k$ short lived states through these avoided crossings or sometimes through avoided crossings which can be related to secondary island chains with higher $q$ than those marked. So in Fig. 19 the two (couples of) crossings which are not marked are those between $k=1$ and $k=9$ and between $k=2$ and $k=10$; both can be reconducted to the $q=8$ secondary island chain but only by analogy with the cases studied, since the crossings happen early in the $k=9$ and $k=10$ curves and their WKB quantization is not yet the resonance one (i.e. depending on the quantum number $k)$. The $(q=8)$ crossing between $k=2$ and $k=10$ appears again as significant in Fig. 20 but in Fig. 21 the transfer of probability at that crossing has dwindled to almost nothing and the only significant crossings are the marked ones. Finally in Fig. 22, corresponding to a case where the classical principal primary resonance zone is almost completely chaotic at the peak of the pulse, the marked crossings are again the first relevant crossings and the only ones to cause significant transfer of probability; but several other crossings cause some (minor) transfer of probability at the peak of the pulse. The most important of these latter crossings are related to classical secondary "fractionary" resonances: for example the crossings marked by crosses (from top to bottom, between $k=0$ and $k=9$, between $k=1$ and $k=10$ and between $k=1$ and $k=11$ ) have $p=2$. There also appears to be some overlap of significant crossings each involving the same level but related to different classical resonance zones (for example the crossings between $k=1$ and $k=5$ and between $k=1$ and $k=11$ in the bottom right corner of the figure); this is by some authors thought to be a sign of an analogous overlap of the related classical resonances and therefore an 
expression in quantum dynamics of local classical chaos [38]. To emphasize the step-like character of the evolution of the $k=0-3$ states, I give in Table I their population loss at each of the relevant crossings.

\section{DISCUSSION}

We have thus shown the relationship between some strictly quantum processes (avoided crossings) and classical structures; but why do isolated avoided crossings so well simulate the classical behaviour in the cases we studied? To understand this we should try and get an idea of the circumstances under which the classical and quantum dynamics can be expected to result in similar evolutions of the observables.

Let us start considering the system at a given microwave field strength; when can we say that the properties of the quantum system reflect the classical resonance structure? Away from any crossing region related to a given secondary classical resonance, the quantum system is by definition not affected by that classical resonance zone. Only for values of $F$ falling within one such crossing region the quantum eigenstates reflect the classical resonance region, as we have seen. If the crossing regions related to the classical resonance zone are well spaced, it will therefore be only by a chance choice of $F$ that the quantum dynamics will be able to "feel" the classical resonance zone. Only when the crossing regions related to the classical resonance zone overlap this element of chance is lost.

If we look at Figure 1 we see that there is hardly any overlap of the crossing regions for any of the $p / q$ ratios marked in the figure; unless $F$ is carefully chosen we therefore expect the fixed microwave field strength quantum dynamics not to be affected by the classical secondary resonance zones for our choice of $n_{0}$; in other terms: we cannot be sure of having any quantum state reflecting (mimicking) the classical secondary resonance structures. This is essentially [39] the base of the objections which have been raised to a classical interpretation of the steps [32], notwithstanding the good agreement of classical and quantum ionization probabilities. I disagree on the ground that arguments for an atom interacting with a constant amplitude microwave field are of limited relevance to the present pulsed system: since the microwave field strength $F(t)$ changes during the pulse, the system in general meets not just one but many avoided crossings related to a single classical resonance (see the examples in Figs. 19-22). Most of these crossings are between completely different states (e.g., for the $q=6$ classical resonance, between the states $k=0$ and $k=6$, between the $k=1$ and the $k=7$ ones and so on ...) and each of these avoided crossings is a potentially very quantum event as we do know that, when a crossing is met by the system twice (once during the rise and the second time during the fall of the pulse), the different phases accumulated by the two parts of the wavefunction (which split at the first crossing) cause interference at the second crossing, resulting in Stueckelberg-like oscillations in the population of the quasienergy states as a function of $F^{\max }$ [40]. We do not see any trace of this quantum behaviour. I believe the cause of this to be twofold. First of all, for the current value of the pulse length $T$, the separatrix crossing event early in the pulse almost immediately significantly spreads out the population on states with resonance quantum number up to $k=3$ as can be seen in Figures 19-22 [41]. This means that, even if there were Stueckelberg oscillations, the ionization signal vs. $F^{\max }$ would be the sum of oscillations due to the couples of crossings met by all four these states, each of the four states meeting a sequence of them. The period of each of these oscillations depends on the phase difference accumulated by the two crossing states between the crossings, the amplitude on the crossing width (and the speed of the crossing) and the phase on the $F^{\max }$ when the crossing is first met by the system. That these oscillations should sum up to a coherent oscillation, rather than average out would be a rare event indeed. Were this the case in our simulations, we should be able to see some sign of these oscillations in the populations of the single states. Namely we would expect to sometimes see at the avoided crossings in the falling edge of the pulse a return of probability from the high $k$ states to the four initially populated ones $(k=0,1,2$ and 3$)$. While this transfer is visible at the very end of the pulse among the four states themselves, we have no evidence of this happening at the avoided crossings we are considering, as can be seen from Table I. A posible cause (our second reason for quasi-classical behaviour) is the very low population on the high $k$ states at crossings on the falling edge of the pulse: at low peak microwave field strengths only high $q$ (narrow) avoided crossings are met so that the population transfer on the rising edge of the pulse is very small. With increasing peak microwave field strength wider crossings, corresponding to lower $q$ 's, are met and population transfer on the rising edge becomes substantial, but so does also the decay rate of the high $k$ states between the two crossings. The importance of the spread of the initial population can on the other hand be inferred from the comparison made in Ref. [32] between the classical and quantum final ionization probabilities for the two pulse times $T=131$ (our case) and $T=300$ microwave periods. In both cases the agreement increases with increasing principal quantum number $n_{0}$; but a good agreement is reached at lower values of $n_{0}$ for $T=131$. This latter is the case where we expect more primary resonance quantum states to be excited at the crossing of the principal primary island separatrix. In this picture, rather than being traces of Stueckelberg oscillations, the fluctuations of the plateaus of the quantum steps in Fig. 2 are likely due to the variations of population transfer at the avoided crossings during the rising edge of the pulse. For any given $q$, the first crossing to be met involves the $k=0$ state and, when first met, happens at the peak of the pulse, so that the interaction time is long and the population transfer high [40]: we have 
a ionization peak. With increasing peak microwave field strength the interaction time at the crossing decreases and so does the population transfer; but then the $k=1$ state has a crossing with the same $q$ and a new ionization peak appears on the tail of the $k=0$ one, and so on.

\section{CONCLUSIONS}

My quantum simulations show that each of the experimentally observed ionization steps is due to a group of avoided crossings between couples of Floquet states belonging to the same quantum nonlinear resonance. These crossings are in their turn related to the classical secondary resonance island chains surrounding the principal primary resonance, as the quantum and classical resonance conditions are formally the same. Finally, the above island chains are responsible for the steps observed in the classical simulations, as their distruction releases into the globally chaotic phase space region (and therefore to eventual ionization) those parts of the electron ensemble previously trapped within them.

The good agreement between quantum and classical simulations is a consequence of the spread - early during the pulse - of the quantum probability to several resonant Floquet states. This spread allows more than one of the avoided crossings related to each of the secondary island chains to redistribute the electron wavefunction to short lived states, thus contributing to the ionization step corresponding to that island chain.

My study allows us to conclude that classical phase space structures too small to influence (in most cases) the quantum evolution for a constant amplitude microwave field, can -in a pulsed regime- generate interesting quantum dynamics. Constant amplitude arguments to determine their relevance should therefore be used with extreme care, as they can lead to wrong conclusions.

\section{ACKNOWLEDGEMENTS}

The author wishes to thank C. Rovelli, G. Mantica and S. Locklin for useful comments and suggestions and the latter also for the use of his computer for the quantum numerical simulations presented in this paper. Special thanks to J.E. Bayfield as advisor of my Ph. D. thesis, amply quoted in the present paper.

\section{APPENDIX A}

I discuss here the choice of integration parameters for my quantum simulations [16]. Let us start with considerations about the extremes of the truncated basis I used. An occupation probability (at every time during the simulation) of the order of $10^{-10}$ on the lowest two or three states can be considered a sign that the basis lowest level $n_{m i n}$ is small enough. For all of my runs with $n_{0}$ in the range 60 to $72, n_{\text {min }}=30$ satisfied this condition.

For the upper bound the matter is more delicate. The spectrum of atom in the static field alone consists of resonances (bands) whose number is not well determined, as increasingly wide ones exist with energies extending above the classical static field barrier. Even if the free atom states $\psi_{n}$ of which my basis is composed extend to infinity their probability distribution is centered around $\langle z\rangle_{n}=3 n^{2} / 2$ with a spread $\Delta z_{n} \cong n^{2} / 2$. This means that truncating the basis puts us in a "fuzzy box". The potential is therefore a kind of "double well" where not only the width but also the depth of the second well varies with the number of levels considered and the calculated spectrum shows couples of quasi degenerate double well states instead of bands. The narrow separation of the couples of levels - up to just below the classical ionization theshold $E_{\max }=-2 F_{s}^{1 / 2}$ - tells us that in the static field alone there is very little coupling between the two sides of the well (as expected for an asymmetric double well [37, 42]). In my pulsed simulations, I take into account the loss of probability induced by the static field by the substitution in equation (3) of $E_{n}$ with $\left(E_{n}-i \Gamma_{n} / 2\right)$, where the decay factors $\Gamma_{n}$ are given by the (3D) formula from Ref. [43]; this is a rather crude approximation: the decay factors are for the Stark states of the problem and I apply them to free atom states but still using Stark perturbative energies in calculating them. This would place us in trouble with states with Stark energies around the maximum of the atom in static field potential $E_{\max }$, were it not for the sharp increase there of the decay rates from practically zero to practically infinity over a range of two or three states. This makes me confident that the errors connected with the use of the wrong states will concern only a few states close to the ionization border which never in our simulations get significantly populated. The continuum above the static field ionization threshold gives no cause for worry: for most of my simulations the uppermost level is $n_{\max }=221$. This gives us about 60 states above the static field ionization threshold, extending in energy about $5.6 * 10^{-5} a$.u. above that threshold. For the frequencies of my simulations $\left(\omega \lesssim 2.7 * 10^{-6} a . u\right.$.) this span in energy is certainly sufficient; moreover, due to the big decay rates of the states of this "pseudo-continuum", the system will not be able to resolve the individual states. More serious cause of worry is that my procedure actually cuts away pieces of the "double well" states and this might 
cause interference effects altering the final result of the simulation. On the other hand such interference effects should be highly sensitive to changes of the basis and extensive tests show this not to be the case.

When the microwave field is switched on, some indication on the validity of the above approximation can be extracted from the quasienergy curves. Ref. [24] has shown that the quasienergies calculated on a truncated basis are subject to the unphysical constraint that their sum is a constant independent from $F$ but dependent on the number of states in the basis. Whatever our choice of basis, certain curves (at the top of the basis) are going to be completely wrong. Now, with increasing microwave field some of the quasienergy states move up and some down; the wrong states are therefore bound to undergo a series of avoided crossings. Since the coupling among the states increases with the microwave field strength, the wrong curves soon or later interact (have a pronounced avoided crossing) with other curves. The avoided crossings of these other curves for fields above the field at which this interaction takes place therefore become in their turn unreliable and so on, to the point that all the quasienergies of interest bear no resemblance to those of the complete problem [44]. Often it is still possible to extract some information from the "wrong" sections of the curves, but it will be mostly qualitative. There are some rules of the thumb to recognize the completely wrong curves. Levels close to the top of the basis are always suspect. Moreover when the nearest neighbour free atom (or, in our case, atom in static field) energy spacing is more than $\omega \hbar$ one expects [24] the quasienergy curves to bend down and viceversa. Curves which do not follow this pattern are usually suspect. The most reliable way of finding the wrong curves is still, on the other hand, to perform test calculations on different bases. For my calculations the free atom basis used was the same I used for the pulsed simulations. For frequencies of interest the lowest level $n_{\min }=30$ (and those just above it) has no wide avoided crossings and is practically at the same quasienergy over the entire range of field strengths spanned by our simulations. Tests where I included all the states down to $\mathrm{n}=1$ show that all levels with lower quantum numbers behave the same way. Tests where I reduced the upmost level from $n_{\max }=221$ to $n_{\max }=98$ show that, for the microwave frequencies of interest here, the behaviour of the levels near $n_{0}$ are very similar over the range of field strengths spanned by my simulations. On the other hand the avoided crossings of the relevant states with the wrong curves at the top of the basis are very narrow for the bigger basis but become much wider for the smaller basis.

We have seen that the free atom basis somehow functions as a Sturmian one does in absence of a static field, reproducing the resonances above the classical static field ionization threshold; but we have also seen that truncation of this basis means the presence of an artificial second potential well with unphysical finite number of discrete states in it. One should therefore wonder about the impact of these levels on the levels of interest. Curves corresponding to states deep in the second "well" tend to have very little interaction with any other state and higher second "well" states tend to have significant avoided crossings only with each other (avoided crossings wide enough that for our pulse lengths they are not crossed diabatically and some population exchange takes place [40]). For the range of microwave field strengths of interest, the only "second well" states having (significant) avoided crossings influencing the "bound states" we are interested in are those close to the classical static field ionization threshold.

Another parameter which can create serious problems is the integration step $\Delta t$ : it must be small enough so that the spurious frequencies $2 \pi k / \Delta t$ induced by the time discretization [15] do not cause transitions between the basis states. Approximating the basis upper limit with the free atom continuum, this means that any $\Delta t<4 \pi n_{m i n}^{2}$ is sufficient to inhibit spurious transitions even between the very little populated extremes of the basis. For $n_{m i n}=30$, the above condition is $\Delta t<10^{4} a . u$. For most of my simulations $\Delta t=5 \cdot 10^{3} a . u$..

\section{APPENDIX B}

\section{An evaluation of the birth and growth of the secondary island chains using the classical pendulum approximation for the principal primary resonance island [16]}

When $F(t)$ is a constant, one procedure often used to study the Hamiltonian (1) is to locally approximate it with an integrable one around any resonant action $I_{r}$ defined by $\omega I_{r}^{3} /\left(1-3 F_{s} I_{r}^{4}\right)=s$ where $s$ is an integer [17]. The resulting Hamiltonian is that of a pendulum describing the slow motion inside the resonance island; for the principal primary resonance $s=1$, it reads

$$
H^{(2)}=-\frac{\beta x^{2}}{2}+\alpha \cos \varphi
$$

where

$$
\begin{gathered}
\beta=3\left(1+F_{S} I_{r}^{4}\right), \quad \alpha=0.325 F_{0}, \\
\varphi=\theta-\omega t,
\end{gathered}
$$


and

$$
x=\frac{I}{I_{r}}-1
$$

The action angle variables of the Hamiltonian (B1) itself are well known. For rotating motion outside the separatrix the (rescaled) action and angle are [45]

$$
\begin{array}{r}
J=\frac{4}{\pi}\left(\frac{\alpha}{\beta}\right)^{1 / 2} \frac{\mathbf{E}(R)}{R}, \\
\varphi_{J}= \pm \pi \frac{F(\varphi / 2, R)}{\mathbf{K}(R)}
\end{array}
$$

where

$$
R=\left(\frac{2}{1-H / \alpha}\right)^{1 / 2}=\frac{1}{|\sin (\Phi / 2)|}
$$

$\Phi$ being the (half) amplitude in $\varphi$ of the oscillations. $\mathbf{E}$ and $\mathbf{K}$ are complete elliptic integrals [45], $F$ is the incomplete elliptic integral corresponding to $\mathbf{K}$, and the $+\operatorname{sign}$ refers to $x>0$. Analogously - for librating motion inside the separatrix - [45]

$$
\begin{gathered}
J=\frac{8}{\pi}\left(\frac{\alpha}{\beta}\right)^{1 / 2}\left[\mathbf{E}(1 / R)-\frac{\left(R^{2}-1\right) \mathbf{K}(1 / R)}{R^{2}}\right], \\
\varphi-J= \pm \pi \frac{R F(\varphi / 2, R)}{2 \mathbf{K}(1 / R)}
\end{gathered}
$$

The frequency of motion in this latter case, known as "slow motion frequency" [46], is:

$$
\Omega \equiv\left|d \varphi_{J} / d t\right|=\left|\partial H^{(2)} / \partial J\right|=\pi(\alpha \beta)^{1 / 2} / 2 \mathbf{K}(1 / R)
$$

which is maximum for $1 / R=0$ where it becomes the usual "small oscillations" frequency

$$
\Omega_{\max }=(\alpha \beta)^{1 / 2} .
$$

Since there is for every $F$ a maximum slow frequency $\Omega_{\max }$, the resonance condition for a secondary resonance with rotation number $p / q$ can be verified only if

$$
\Omega_{\max }>p \omega / q=p\left(1-3 F_{S} I_{r}^{4}\right) / q I_{r}^{3} .
$$

The above condition for the existence of a $p / q$ secondary resonance zone can be written in the form of a condition on the microwave field strength:

$$
F I_{r}^{4}>F_{p / q} I_{r}^{4} \equiv \frac{\left(1-3 F_{S} I_{r}^{4}\right)^{2} p^{2}}{0.975 q^{2}\left(1+F_{S} I_{r}^{4}\right)} \cong \frac{\left(1-7 F_{S} I_{r}^{4}\right) p^{2}}{0.975 q^{2}}
$$

For $F<F_{p / q}$ the secondary resonance zone does not exist and it appears for $F=F_{p / q}$ at the center of the primary resonance zone, $\Omega_{\max }$ being the pendulum libration frequency there. Contrary to the primary resonance zones, it is clear that since $\Omega=\Omega(J, F)$, the secondary resonance zones do move in phase space. To see how they move we can combine (for $F>F_{p / q}$ ) the $p / q$ resonance condition (B6) with eq. (B4) expanded in powers of $1 / R=|\sin (\Phi / 2)|$; this gives us the distance in $\varphi$ of the secondary island chain from the primary fixed point:

$$
\Phi \cong\left(8 \Delta F / F_{p / q}\right)^{1 / 2}
$$

where $\Delta F=F-F_{p / q}$. We thus see that for every $p / q$ the resonance starts for $F=F_{p / q}$ - called the $p / q$ bifurcation field - at the fixed point itself $(\Phi=0)$ and then moves outwards toward higher $\Phi$ 's with increasing $F$.

Eqs. (B7) and (B8) have been obtained in pendulum approximation, we therefore expect them to work only up to moderate fields; their failure for low $q$ integer resonances - which appear only at high microwave fields - is particularly evident for eq. (B8) where the exponent $1 / 2$ does not fit the numerical results for $q \leq 4$ [13]. Another evaluation of the bifurcation fields $F_{p / q}$ can be obtained [16] from a stability analysis of the Kepler map: an approximate map 
for the classical dynamics derived in Ref. [17]. In the map the fixed point at the center of the principal primary resonance becomes unstable after the $p / q=1 / 2$ bifurcation point. This is confirmed by my numerical simulations for the complete system which show no trace of the primary stable fixed point above the $F_{1 / 2}$ bifurcation point. The fractional deviation of the Kepler map bifurcation points from the numerical ones is approximately constant: they are always $25 \%$ below the numerical ones, while the pendulum approximation bifurcation points are always above the numerical ones and their deviation increases from about $5 \%$ for $q=7$ and 6 to about $128 \%$ for $q=2$. The introduction of a static field $F_{S}=8 \mathrm{~V} / \mathrm{cm}$ reduces in our case the bifurcation points by about $20 \%$. Again, in all cases the results in pendulum approximation are higher than the numerical ones and the results from the Kepler map lower; but while the deviation of the pendulum approximation results increases from about $15 \%$ at $q=6$ to about $150 \%$ at $q=2$, the deviation of Kepler map results from the numerical ones remains about $15 \%$ from $q=6$ to $q=2$. Since in absence of a static field the deviation was about 25\%; this suggest that the linear approximation of the static field I used is an over-evaluation.

\section{2. quantum pendulum approximation and WKB quantization [16]}

Following Ref. [25] and [36], the quasienergies of the Hamiltonian (1) can be approximated by

$$
\varepsilon_{k}^{(1)}=\left(E_{R}+W_{k}\right)(\bmod \omega \hbar) .
$$

where $E_{R}$ is the eigenvalue of the (classically) resonant state $\mid R>$ of the Hamiltonian for the atom in the static field alone; and $W_{k}$ denote the eigenvalues of the matrix $H_{n, m}^{(1)}$ defined as follows:

$$
\begin{array}{r}
H_{n, n}^{(1)}=\left[E_{n}-E_{R}-\omega(n-R)\right], \\
H_{n, n+1}^{(1)}=F\langle R|z| R+1\rangle / 2
\end{array}
$$

While the diagonalization of the Floquet operator gives us no natural ordering of the quasienergies, the energies $W_{k}$ can be ordered. A descending order in energy with $k$ starting from zero allows us to identify the index $k$ as the quantized version of the pendulum action $J$. Moreover, since eq. (B9) gives energies folded into the first Brillouin zone, the level crossings appearing as a consequence of this folding are actual crossings. Eq. (B9) has been shown to give for quantum numbers close to $R$ quasienergy curves remarkably similar to those of the full problem [36] for $n_{0}=60$ and rescaled fields up to about $F_{0}=0.04$. I have numerically calculated the "quasienergies" (B9) for the parameters of Fig. 1; the matrix elements $H_{n, m}^{(1)}$ between the Stark states were calculated by projecting the Stark states on the free atom basis $n \in[33,94]$.The result is shown as the dotted curves in Fig. 23. For comparison, I also plotted - as big dots connected by dark lines - the result of a WKB quantization of easily calculated approximations of the constant action $J_{0}$ curves of energy versus microwave field strength $F_{0}$ implicitly defined by eqs. (B3) and (B2). The approximations made were the following: The zero action curve is the energy of the fixed point: $H=\alpha$. Near $J_{0}=0$ we can expand $\mathbf{K}(1 / R)$ and $\mathbf{E}(1 / R)$ in powers of $1 / R$ (to the fourth order [45]) and then solve for $H$ the resulting approximation of eq. (B3). For a given action $J_{0}$ this approximation will get better and better for increasing $\alpha$ : in the high field limit equidistance in action will therefore mean equidistance in energy and the system will behave essentially like a harmonic oscillator with frequency $\Omega=(\alpha \beta)^{1 / 2}$. A similar procedure is used in the opposite limit $(\alpha \rightarrow 0)$ starting from eq. (B2). At the separatrix $(H=-\alpha)$, there is a discontinuity in $J: J=4(\alpha / \beta)^{1 / 2} / \pi$ outside and $J=8(\alpha / \beta)^{1 / 2} / \pi$ inside; but, from both sides, approaching the separatrix, $d H / d \alpha$ tends to -1 so that the $H(\alpha)$ curves join smoothly $\left(C^{1}\right.$ at least). Since their slope at that point is that of the separatrix itself, we also know we have an inflection point there. Finally the curve $H(\alpha)$ has a minimum given by the condition $2 \mathbf{E}(1 / R)=\mathbf{K}(1 / R)$ which solved with the help of the numerical tables [47] gives us $\mathbf{K}(1 / R)=2.3211$ and $J=8(\alpha / \beta)^{1 / 2}(-H / a) \mathbf{E}(1 / R) / \pi \cong 1.1128(3 \alpha / \beta)^{1 / 2}$. With the above approximations and using the WKB $n$-quantization outside the classical resonance zone and the $k$-quantization inside, we have:

Low field behaviour:

$$
\begin{aligned}
\frac{\varepsilon_{k}}{\omega} & \cong\left[\frac{-\left|H_{0}\right|-\frac{\alpha^{2}}{8\left|H_{0}\right|}}{\omega}\right] \bmod (1) \\
& =\left[\frac{\varepsilon_{k}(0)}{\omega}-\frac{\left(\frac{0.325 F_{0} I_{r}^{2}}{n_{0} \omega_{0}}\right)^{2}}{\frac{8\left|\Delta_{k}(0)\right|}{\omega}}\right] \bmod (1),
\end{aligned}
$$


Minimum:

$$
\begin{aligned}
F_{0} & =\frac{1+F_{s}^{0}\left(I_{r} / n_{0}\right)^{4}}{0.325}\left[\frac{(k+1 / 2) n_{0}^{2}}{1.1128 I_{r}^{3}}\right]^{2} \\
\frac{\varepsilon_{k}}{\omega} & \cong\left[\frac{\varepsilon_{r}}{\omega}-0.6522 \frac{\alpha}{\omega}\right] \bmod (1) \\
& =\left[\frac{\varepsilon_{r}}{\omega}-0.6522 \frac{0.325 F_{0} I_{r}^{2}}{n_{0} \omega_{0}}\right] \bmod (1)
\end{aligned}
$$

High field behaviour:

$$
\begin{aligned}
\frac{\varepsilon_{k}}{\omega} \cong\left\{\frac{\varepsilon_{r}}{\omega}+\frac{\alpha}{\omega}\left[9-8\left(1+\frac{J_{0}(3 / \alpha)^{1 / 2}}{4}\right)^{1 / 2}\right]\right\} \bmod (1) \\
\\
=\left\{\frac{\varepsilon_{r}}{\omega}+\frac{0.325 F_{0} I_{r}^{2}}{n_{0} \omega_{0}}\left[9-8\left(1+\frac{\mathcal{R}}{4}\right)\right]^{1 / 2}\right\} \bmod (1)
\end{aligned}
$$

where

$$
\mathcal{R}=\frac{(k+1 / 2) n_{0}^{2}}{I_{r}^{3}}\left(\frac{3\left[1+F_{0}^{s}\left(I_{r} / n_{0}\right)^{4}\right]}{0.325 F_{0}}\right)^{1 / 2} .
$$

In Fig. 23 the resonant action is $I_{r}=69$ and the zero microwave field parameters $\varepsilon_{k}(0)$ and $\left|\Delta_{k}(0)\right|=-\Delta_{k}(0)=$ $W_{k}(0)=\varepsilon_{r}-\varepsilon_{k}(0)$ used in the construction are given in table II (in atomic units). The agreement is quite good both at high and low fields; the shift of the high field curves increases with $k$ but for each of these curves it appears to slowly decrease with increasing field. The inflection points, which classically are right at the separatrix, are at the center of the transition region between the two quantizations: to their left the states are essentially modulated Stark states while to their right they take the character of resonant states. In our semiclassical treatment we can therefore only say that they fall somewhere between the $n$-quantized point and the $k$-quantized one (circled points connected by segments in Fig. 23)

\section{a. Semiclassical scaling of the quasienergy curves [16]}

Reference [48] suggests an approximate semiclassical scaling law:

$$
\varepsilon_{n}(F) / \omega^{2 / 3} \approx f_{n \omega^{1 / 3}}\left(F / \omega^{4 / 3}\right)
$$

where the function $f$ depends only on classically rescaled quantities. Eq. (B12) connects quasienergy curves at different frequencies: to get the same function $f_{n \omega^{1 / 3}}$ for a different quantum number $n$, $\omega$ has to be changed. The agreement of my classical pendulum approximation evaluations with the quantum curves confirms for the cases studied this hypothesis, but only up to a certain point: while my evaluation for the low field behaviour, the top equation in (B11), scales exactly according to eq. (B12), the evaluations for the minimum and the high field behaviour depend not only on $n$ but also on $(k+1 / 2) / n$, as can be seen from the two bottom eqs. in (B11).

[1] M. Berry, "Regular and irregular motion" in Am. Inst. Phys. Conf. Proc. No.46, S. Jorna Ed.

[2] A. R. Bishop, R. E. Ecke, and R. Mainieri, Quantum Complexity in Mesoscopic Systems, North Holland, 1995; and G. Casati and B. Chirikov, Quantum Chaos: Between Order and Disorder, Cambridge Univ. Press, 1995.

[3] See e.g. S. D. Frischat and E. Doron, Phys. Rev.E57 1421 (1998); O. Brodier, P. Schlagheck, and D. Ullmo, Phys. Rev. Lett. 87, 064101 (2001); C. Eltschka and P. Schlagheck, Phys. Rev. Lett. 94, 014101 (2005); A. Shudo, Y. Ishii, and K. S. Ikeda, J. Phys. A 35, L225 (2002) and references therein.

[4] See e.g. F. B. Dunning, J. J. Mestayer1, C. O. Reinhold, S. Yoshida, and J. Burgdoerfer, J. Phys. B 42, 022001 (2009); A. Buchleitner, D. Delande, and J. Zakrzewski, Phys. Rep. 368, 409 (2002); H. Maeda and T. F. Gallagher, Phys. Rev.A75, 033410 (2007) and references therein.

[5] See e.g. E. Shchekinova,C. Chandre, and T. Uzer, Phys. Rev.A74, 043417 (2006).

[6] R. V. Jensen, M. M. Susskind, and M. M. Sanders, Phys. Rep. 201, 1 (1991). 
[7] E. J. Galvez, B. E. Sauer, L. Moorman, P. M. Koch, and D. Richards, Phys. Rev. Lett. 61, 2011 (1988).

[8] K. Dietz, J. Henkel, and M. Holthaus, Phys. Rev. A45, 4960 (1992).

[9] J.D. Meiss, Rev. Mod. Phys. 64, 795 (1992).

[10] Another example of phase space transport process for which the connection between classical and quantum aspects have been investigated is the scattering of atoms by a laser standing wave; see e.g. R. Graham, M. Schlautmann and P. Zoller, Phys. Rev. A45, R19 (1992); A. R. Kolovsky and H. J. Korsch, Phys. Rev. A55, 4433 (1997) and Phys. Rev. A57, 3763 (1998).

[11] J. E. Bayfield and D. W. Sokol, Phys. Rev. Lett. 61, 2007 (1988).

[12] Y.-C. Lai, C. Grebogi, R. Blumel, and M. Ding, Phys. Rev. A45, 8284 (1992).

[13] J.E. Bayfield, S.Y. Luie, L.C. Perotti, and M.P. Skrzypkovski, Phys. Rev. A53, R12 (1996).

[14] See e.g.: J.E. Bayfield, S.Y. Luie, L.C. Perotti, and M.P. Skrzypkovski, Physica B83, 46 (1995).

[15] G. Casati, B.V. Chirikov, D.L. Shepelyansky, and I. Guarneri, Phys. Rep. 154, 77 (1987).

[16] L. Perotti; Ph. D. Thesis, Un. of Pittsburgh (1996).

[17] G. Casati, I. Guarneri, and D.L. Shepelyansky, IEEE J. Quantum Electronics 24, 1420 (1988).

[18] G. Radons and R. E. Prange, Phys. Rev. Lett. 61, 1691 (1988).

[19] M. V. Berry, N. L. Balazs, M. Tabor and A. Voros; Ann. Physik 122, 26 (1979).

[20] G. Casati and L. Molinari, Suppl. Progr. Theor. Phys. 98, 287 (1989).

[21] K. Yaijma, Comm. Math. Phys. 87, 331 (1982).

[22] H.P. Breuer, K. Dietz, and M. Holthaus, J. Phys. B22, 3187 (1989).

[23] J. Von Neumann and E. Wigner, Phys. Z. 30, 467 (1929).

[24] H.P. Breuer and M. Holthaus, Z. Phys. D11, 1 (1989).

[25] G.P. Berman and G.M. Zaslavsky, Phys. Lett. A61, 295 (1977).

[26] M. Holthaus; Chaos, Solitons \& Fractals 5, 1143 (1995).

[27] A. Buchleitner, These de Doctorat de l'Université Pierre et Marie Curie, Paris 6 (1993); D. Delande and A. Buchleitner, Adv. At. Mol. and Opt. Phys., 34, 85 (1994), and D. Delande, J. Zakrzewski and A. Buchleitner, Europhys. Lett. 32, 107 (1995).

[28] K. Husimi, Proc. Phys. Math. Soc. Jpn. 22, 264 (1946).

[29] R.V. Jensen, M.M. Sanders, M. Saraceno, and B. Sundaram, Phys. Rev. Lett. 63, 2771 (1989).

[30] If the evolution is adiabatic, each point remains at the same resonance action $J$ and since new actions keep being added by the growth of the resonance zone, the span in angle of the ensemble decreases. If instead the evolution is diabatic, the ensemble is not "squeezed" but does not expand either. Non-adiabatic evolution will be intermediate between the two, resulting in partial "squeezing". Note that since the resonance action on an invariant (resonance) curve is the area bounded by the invariant curve itself divided by $2 \pi$, "squeezing" a resonance torus in the angle variable will cause it to expand in the action variable. The same conclusion is reached by applying Liouville's theorem to the area included within a resonance invariant curve.

[31] Trapping in moving phase space structures has been given the name of "bucket dynamics"; see e.g. J. M. Yuan and W. K. Liu, Phys. Rev. A57, 1992 (1998).

[32] D. Richards, J. Phys. B29, 5237 (1996).

[33] This also explains a feature of our classical ionization vs. $\omega_{0}^{\prime}$ curves at constant $F_{0}^{\max }$ : all curves showed a marked dip at $\omega_{0}^{\prime} \cong 1$, but the position in $\omega_{0}^{\prime}$ of the minimum of this dip depended on $F_{0}^{\max }$ in an oscillatory manner. The point is that the higher is $\omega_{0}^{\prime}$, the higher is $F_{0}^{\max }$ corresponding to a given $F_{r}^{\max }$. This means that in a plot at constant $F_{0}^{\max }$ it is possible for a secondary island chain to be destroyed at low frequencies but not at high ones, resulting in lower ionization probabilities for high frequencies and therefore in the curve having its minimum at $\omega_{0}^{\prime}>1$. This will happen for those values of $F_{0}^{\max }$ close to the onset of a step; for $F_{0}^{\max }$ close to the center of a plateau we instead expect the shape of the dip to be determined principally by the primary resonance. As expected, the minima of the dips are at $\omega_{0}^{\prime}=1$ except near the steps where they are at $\omega_{0}^{\prime} \gtrsim 1$.

[34] I. Percival and D. Richards, Introduction to Dynamics, Cambridge Un. Press (1982).

[35] G.A. Voth, J. Phys. Chem. 90, 3624 (1986).

[36] L. Sirko and P.M. Koch, Appl. Phys. B60, S195 (1995).

[37] M.J. Davis and E.J. Heller, J. Chem. Phys. 75, 246 (1981).

[38] T. Uzer, D.W. Noid and R.A. Marcus, J. Chem. Phys. 79, 4412 (1983).

[39] In effect the argument in Ref. [32] is that no single island in the secondary chains is big enough to significantly support a quantum state. It is equivalent to requiring quantum multilevel (involving more than two levels at a time) interactions relating to a $p / q$ classical resonance zone [16] and it is a more stringent condition than the one we require, since even in the case of overlapping crossing regions the quantum dynamics may still be a two level one: if $q \neq 1$, the pairs of states coupled by the overlapping crossing zones may have no state in common, even if the crossing regions are related to the same classical resonance zone. As a simple example let us consider the $p / q=1 / 3$ crossing in Fig. 13 : with increasing $F$, the first crossing we encounter is the $\left(k_{1}, k_{2}\right)=(0,3)$ one (just to the right the classical $p / q=1 / 3$ bifurcation field); the following one is the $(1,4)$ one, followed by (out of the picture) the $(2,5)$ one and only then by the $(3,6)$ one which again has $k=3$ as a level taking part in the crossing. To be able to have one quantum multilevel (involving more than two levels at a time) interaction relating to a $\mathrm{p} / \mathrm{q}$ classical resonance zone we therefore need at least $q+1$ crossing regions to overlap at the same $F$. Based on the $q$-fold structure our Husimi functions at a crossing in Figs. 14-18, we prefer to use the size of the whole chain, instead of that of a single island. Moreover, from Figs. 14-18 it is clear that the structure responsible for the steps is not a single secondary island, but the whole chain of them. Finally, we find fault with the argument (often adduced 
in support of considering the area of a single island of the chain) that, since the system (at constant field strength) is time periodic, all the islands of a chain belong to the same vortex tube in the extended phase space $\mathcal{P}=\{I, \theta, H, t \in(0, T)\}$ and therefore only one section (a single island) of the tube should be considered when evaluating how many quantum states (of area $h$ ) the classical chain can support. Our objection is twofold: first, the quantities to compare would in this case be the volume of the vortex tube in $\mathcal{P}$ and $h^{2}$. Second, as pointed out in Ref. [26], quantization of a single vortex tube would result in a $q T$ periodic wavefunction, which is not a Floquet eigenstate.

[40] L.D. Landau, Phys. Z. Sov. 2, 46 (1932); C. Zener, Proc. R. Soc. London A137, 696 (1932); E.C.G. Stueckelberg, Helv. Phys. Acta 5, 370 (1932).

[41] A comparable classical spread can be inferred for example from the first snapshot of Figure 8 where the ensemble is spread over an approximately triangular region of (rescaled) area $A \cong 0.14(2 \pi 0.83) / 2$ corresponding to a spread $\Delta k=n_{0} A / 2 \pi \cong 4$.

[42] E.J. Heller and M.J. Davis, J. Phys. Chem. 85, 307 (1981).

[43] Damburg and Kolosov in Rydberg states of atoms and molecules, R.F. Stebbings and F.B. Dunning eds. , Cambridge (1983).

[44] For a discussion of the problems connected to basis truncation in the calculation of quasienergies see e.g. D. W. Hone, R. Ketzmerick and W. Kohn, Phys. Rev. A56, 4045 (1997).

[45] I.S. Gradshtenyn and I.M. Ryzhik, Table of Integrals, Series, and Products, Academic Press (1980).

[46] G.P. Berman and A.R. Kolovskii, Sov. Phys. Usp. 35, 303 (1992).

[47] M. Abramowitz and I.A. Stegun, Handbook of mathematical functions, Dover Publ. New York (1964).

[48] H.P. Breuer and M. Holthaus, Ann. of Phys. 211, 249 (1991). 


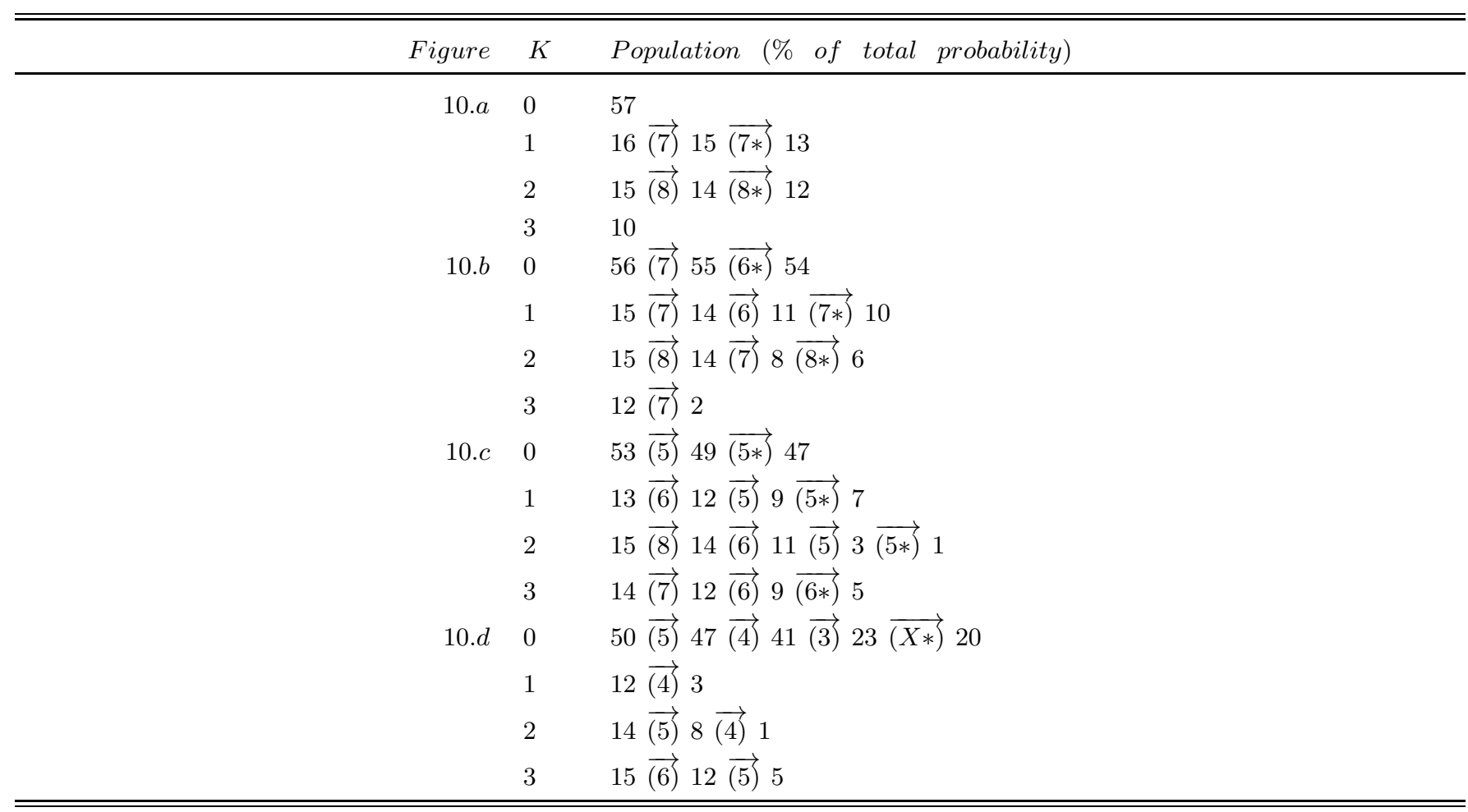

TABLE I: The change of population on the four central resonance states in Figures 19-22 at the relevant avoided crossings. The populations are in percent of the total unit population. The $q$ for each crossing is given in parenthesis, an asterisc follows crossings on the falling edge of the pulse. The $x$ denotes a $p=2, q=9$ crossing.

\begin{tabular}{lllll}
\hline \hline$k$ & $n$ & $E_{k}(0) / \omega$ & $\left|\Delta_{k}(0)\right| / \omega$ \\
\hline 0 & 69 & 0.24370 & 0 \\
1 & 70 & 0.23218 & 0.0115 \\
2 & 68 & 0.20187 & 0.0418 \\
3 & 71 & 0.17168 & 0.0720 \\
4 & 67 & 0.10540 & 0.1383 \\
5 & 72 & 0.06417 & 0.1795 \\
6 & 66 & 0.95055 & 0.2927 \\
7 & 73 & 0.91201 & 0.3310 \\
8 & 65 & 0.73391 & 0.5098 \\
9 & 74 & 0.71738 & 0.5236 \\
\hline \hline
\end{tabular}

TABLE II: Quantum parameters for the semiclassical curves in Fig. 23. (From Ref. [16]) 


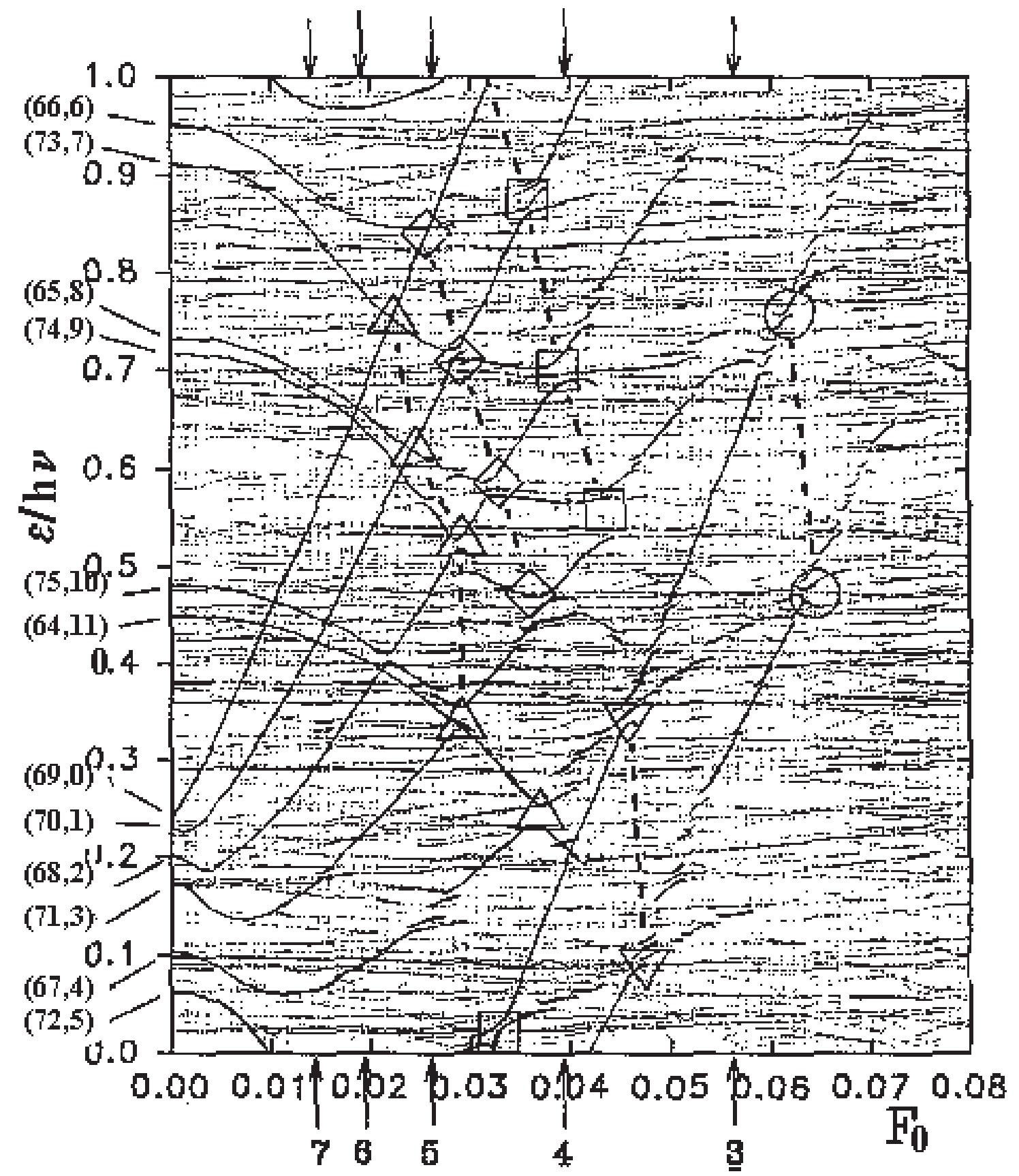

FIG. 1: Quasienergy curves for $n=17.50 \mathrm{GHz}$ and $F_{s}=8 \mathrm{~V} / \mathrm{cm}$ calculated on the basis $n \in$ [30, 221]. The horizontal axis is the microwave field strength rescaled for $n=69$. The darker curves are those related to the $s / r=1$ quantum resonance. The labels $(n, k)$ give the free atom quantum number $n$ and the resonance quantum number $k$. The groups of avoided crossings related to the most visible classical secondary island chains with rotation number $1 / q$ are indicated by the following symbols: $q=3$ circles; $q=4$ down triangles; $q=5$ squares; $q=6$ diamonds; $q=7$ up triangles. To aid the eye, lines are passed through the members of each group. Also, arrows mark the classical numerical bifurcation fields, labeled by $q$. (From Ref. [16]) 


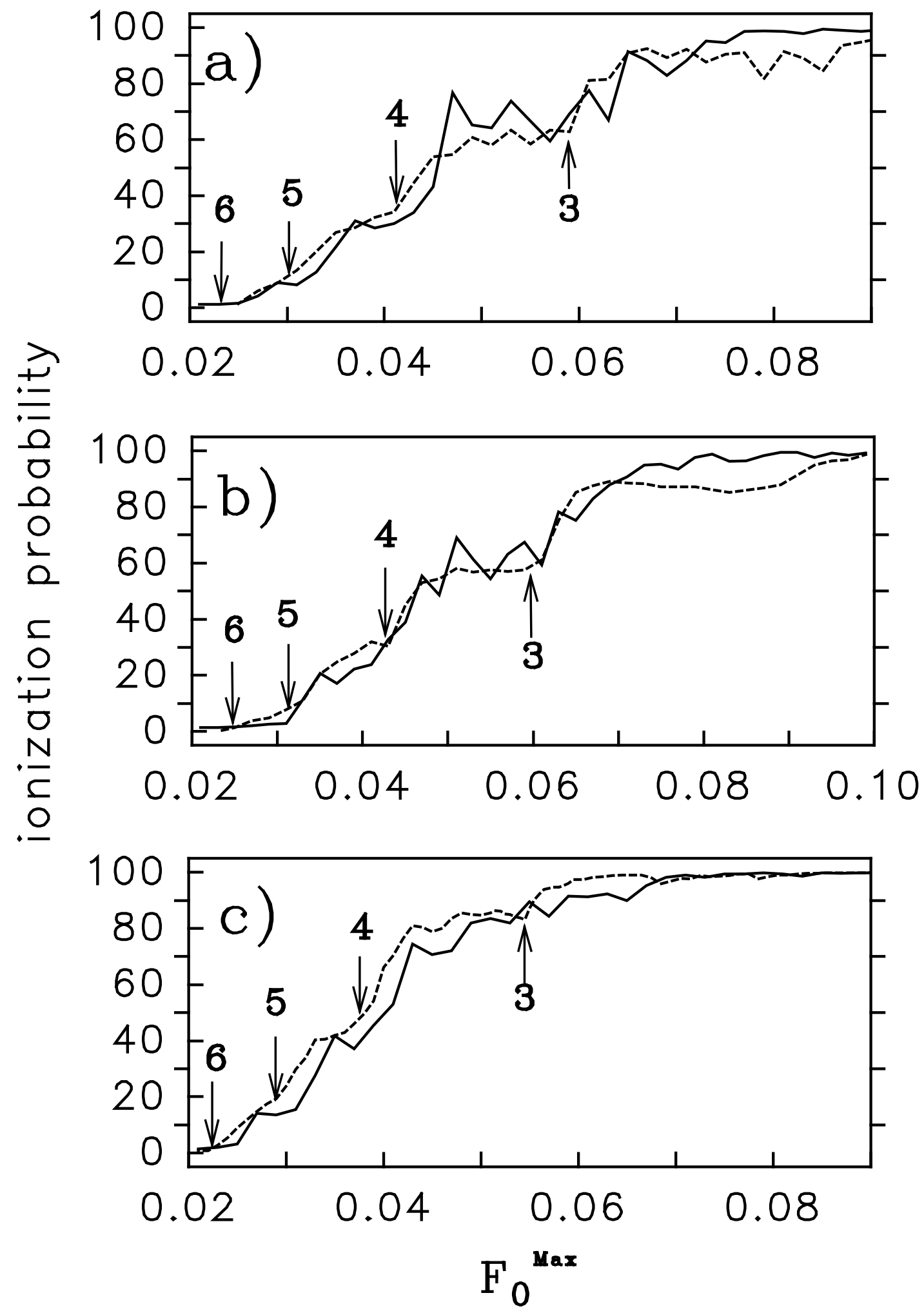

FIG. 2: Classical (dashed lines) and quantum (full lines) steps for a) $\omega_{0}^{\prime}=0.9771$, b) $\omega_{0}^{\prime}=0.9994$ and c) $\omega_{0}^{\prime}=0.927$; $F_{0}^{S}=0.035266$. Classical destabilization fields for secondary island chains are indicated by arrows; they are in all cases very close to the onset of a ionization step. (From Ref. [16]) 


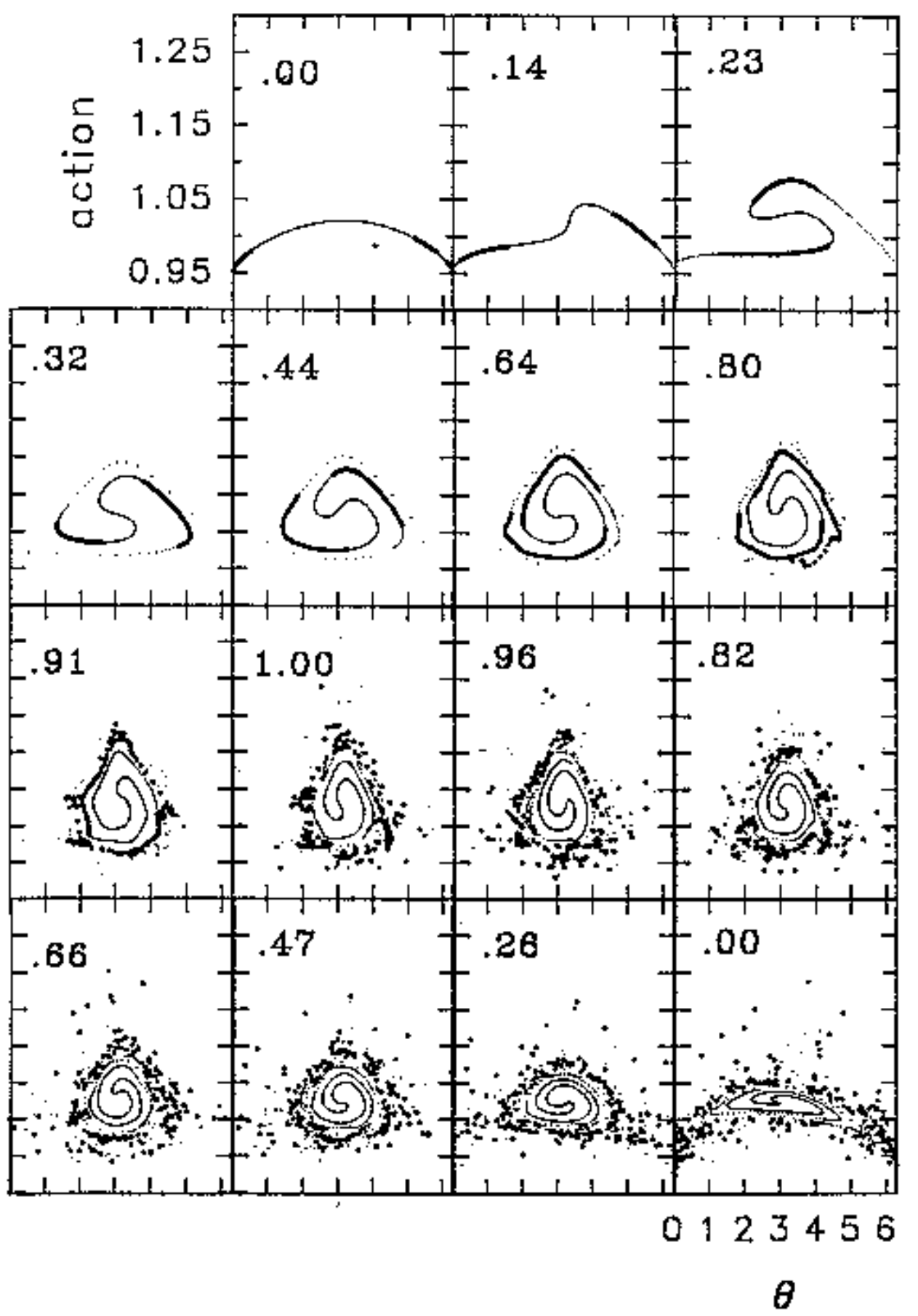

FIG. 3: Snapshots of the evolution of the classical ensemble for the parameter values $\omega_{0}^{\prime}=0.9771, F_{0}^{S}=0.035266$ and $F_{0}^{\max }=0.025$. The ratio of $F_{0}(t)$ to $F_{0}^{\max }$ is indicated in the top left corner of each snapshot. Bigger dots mark the part of the ensemble within the $q=6$ island chain at the peak of the pulse. (From Ref. [16]) 


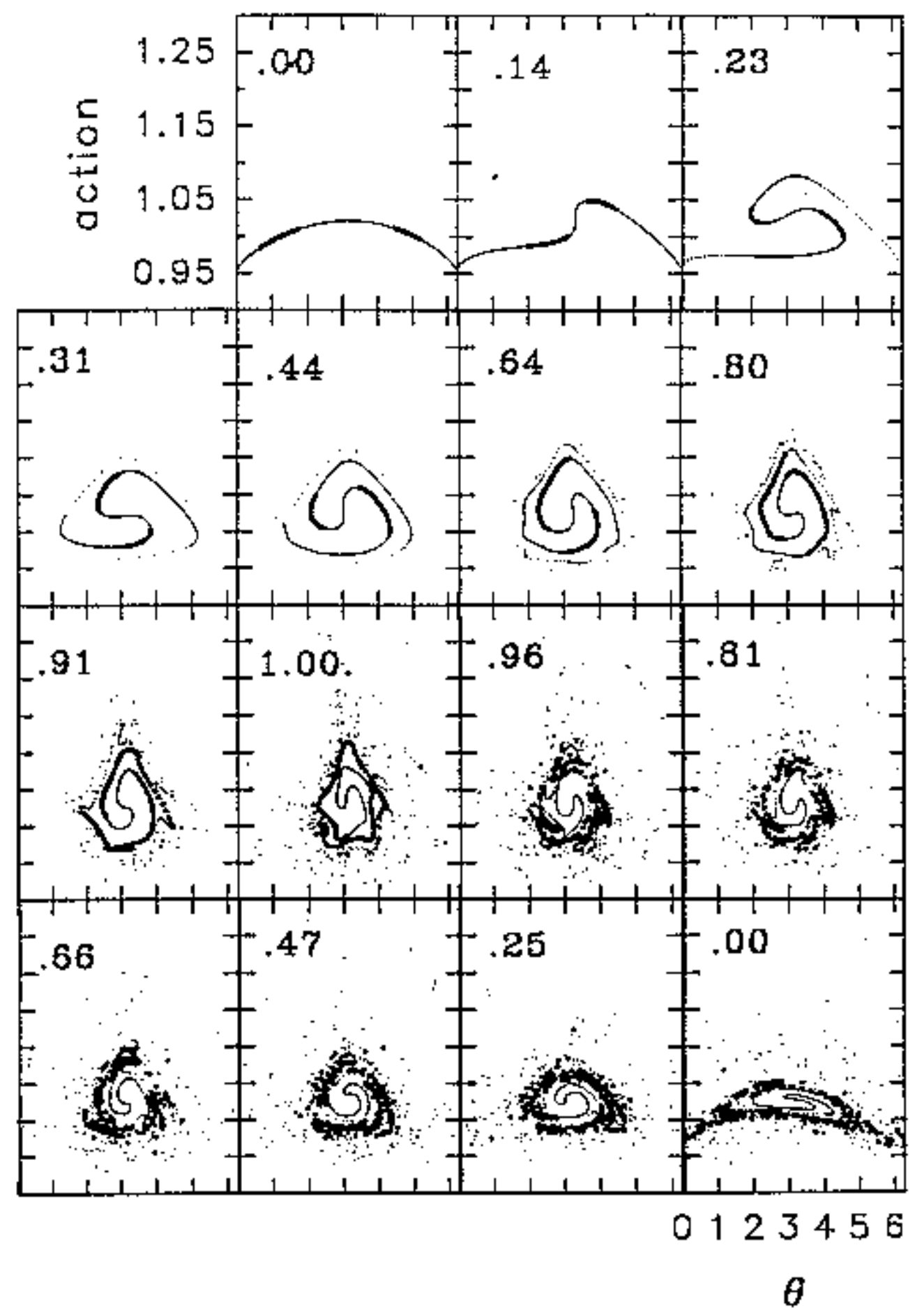

FIG. 4: Snapshots of the evolution of the classical ensemble for the parameter values $\omega_{0}^{\prime}=0.9771, F_{0}^{S}=0.035266$ and $F_{0}^{\max }=0.029$. The ratio of $F_{0}(t)$ to $F_{0}^{\max }$ is indicated in the top left corner of each snapshot. Bigger dots mark the part of the ensemble within the $q=5$ island chain at the peak of the pulse. (From Ref. [16]) 


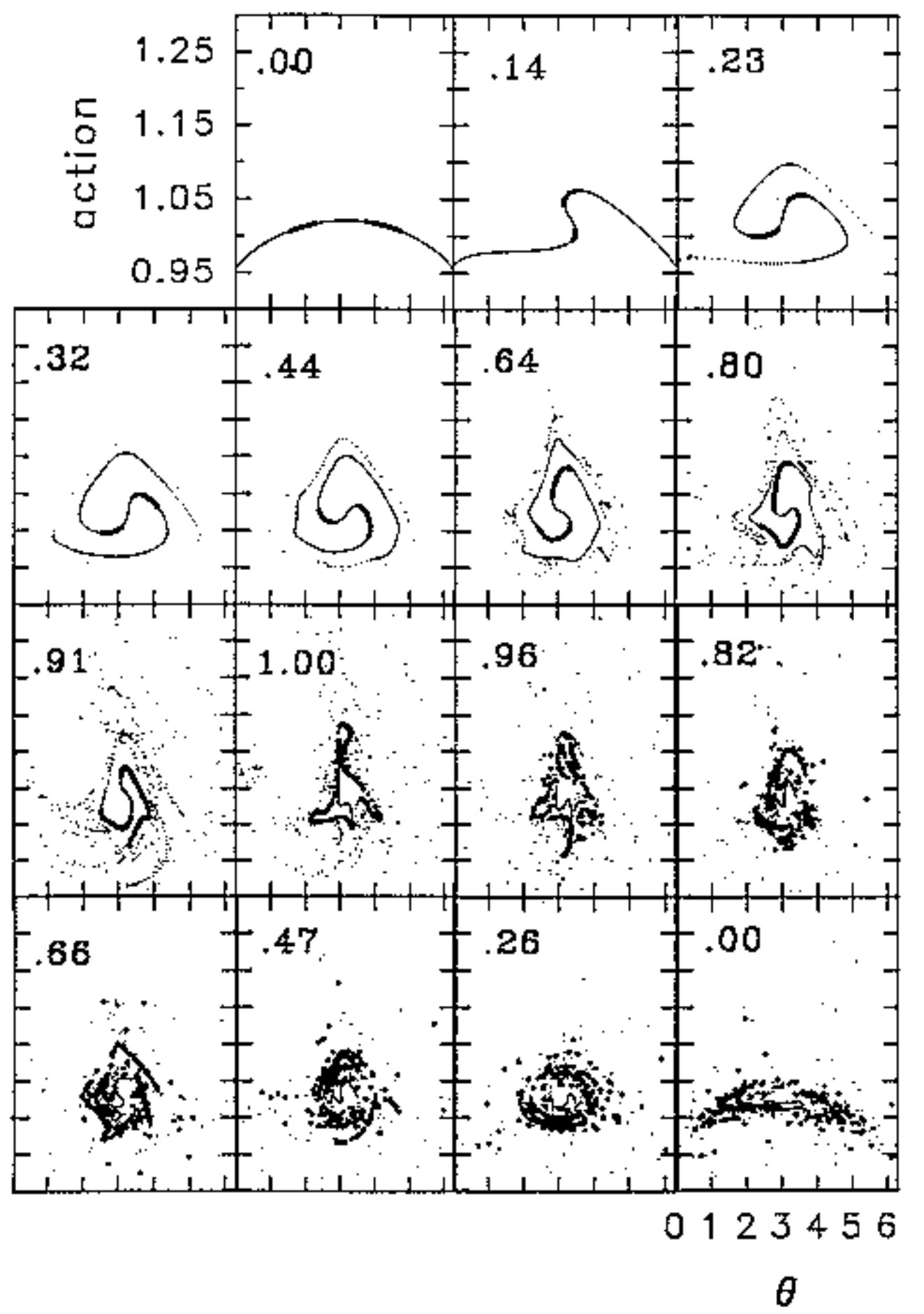

FIG. 5: Snapshots of the evolution of the classical ensemble for the parameter values $\omega_{0}^{\prime}=0.9771, F_{0}^{S}=0.035266$ and $F_{0}^{\max }=0.041$. The ratio of $F_{0}(t)$ to $F_{0}^{\max }$ is indicated in the top left corner of each snapshot. Bigger dots mark the part of the ensemble within the $q=4$ island chain at the peak of the pulse. (From Ref. [16]) 


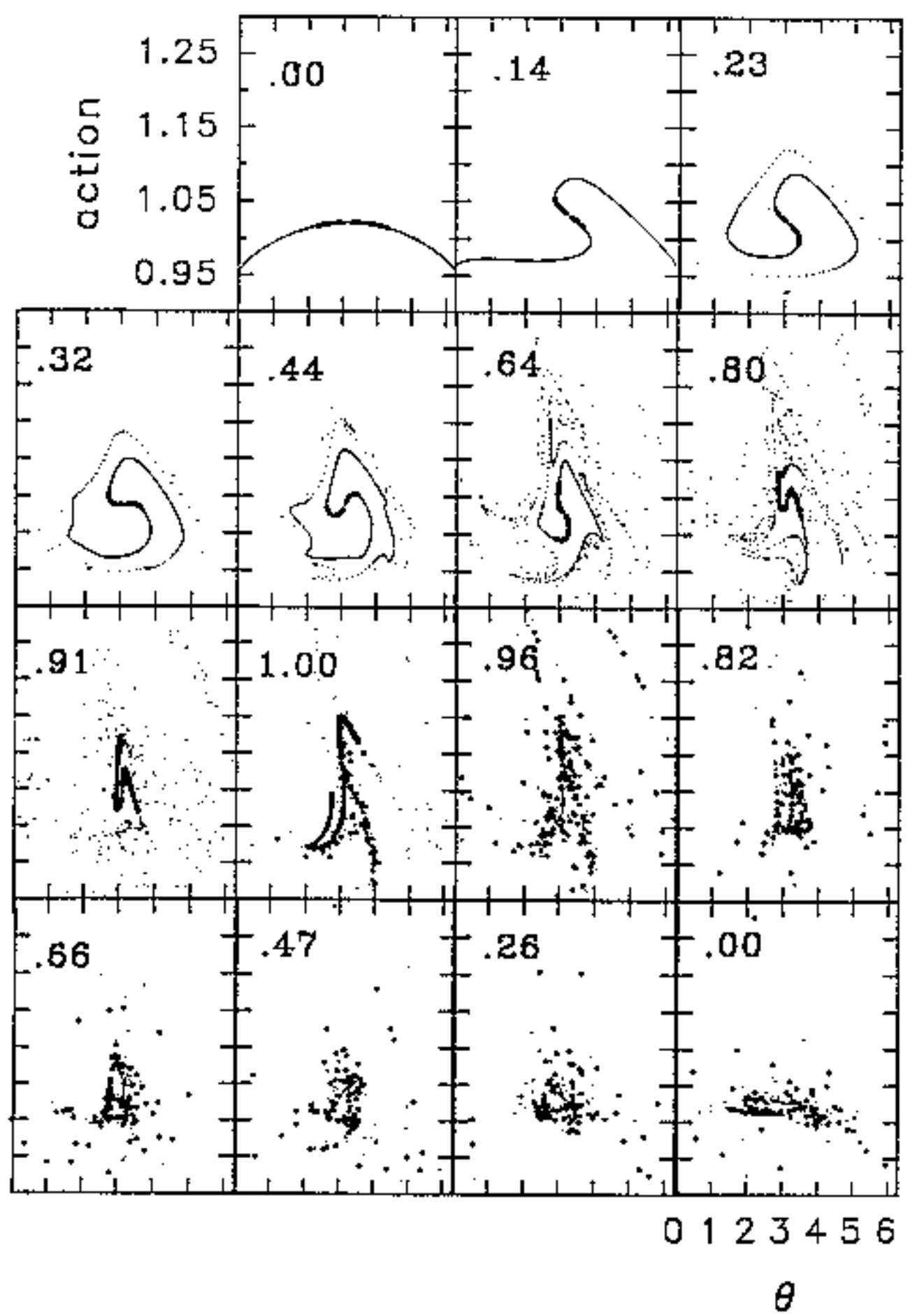

FIG. 6: Snapshots of the evolution of the classical ensemble for the parameter values $\omega_{0}^{\prime}=0.9771, F_{0}^{S}=0.035266$ and $F_{0}^{\max }=0.061$. The ratio of $F_{0}(t)$ to $F_{0}^{\max }$ is indicated in the top left corner of each snapshot. Bigger dots mark the part of the ensemble within the $q=3$ island chain at the peak of the pulse. (From Ref. [16]) 


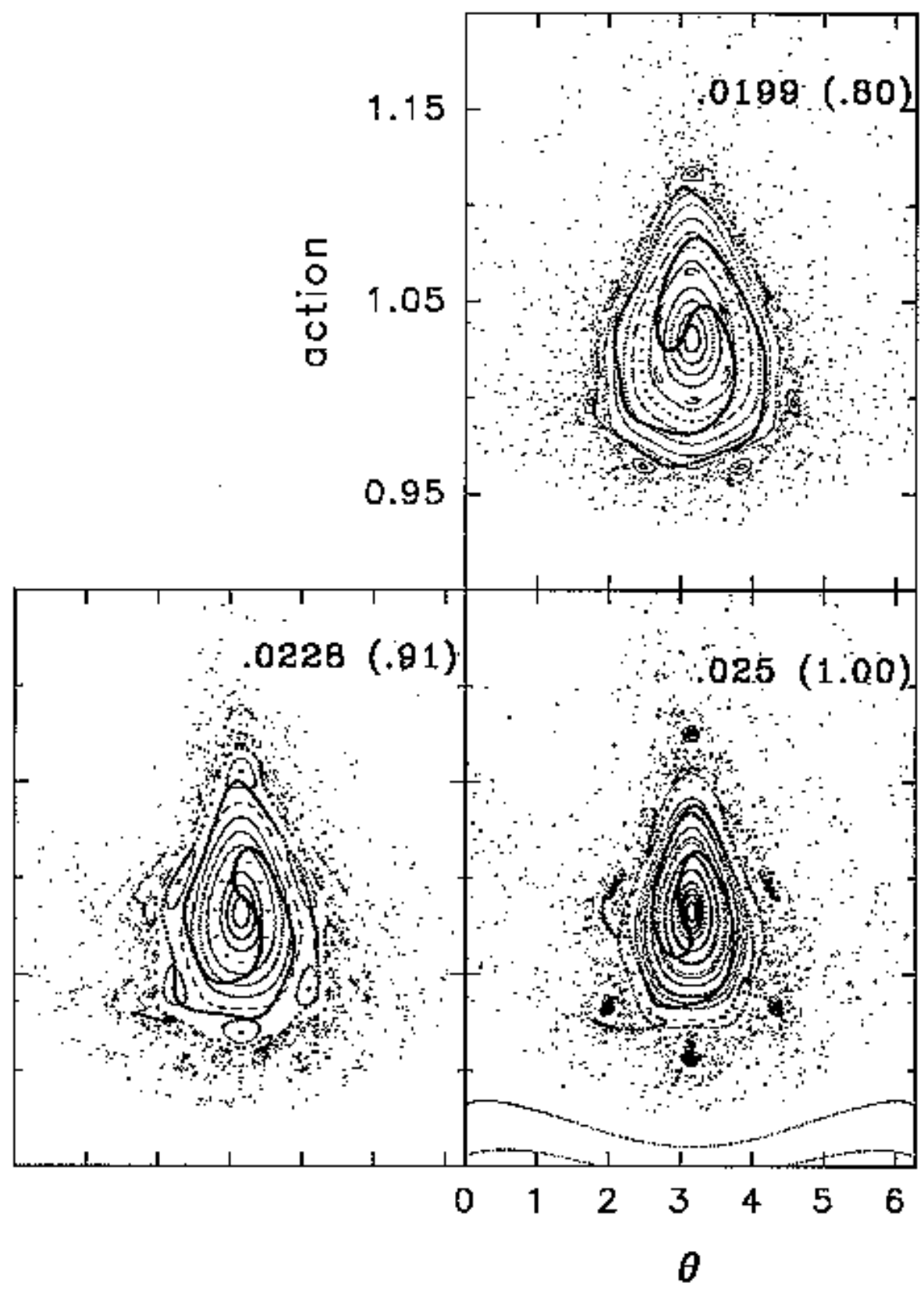

FIG. 7: Comparison of snapshots from Fig. $3\left(F_{0}^{\max }=0.025\right)$ with the surfaces of section calculated at the values of the instantaneous $F_{0}(t) ; F_{0}$ is indicated at the top of each graph, its ratio to $F_{0}^{\max }$ is given in parenthesis. In the first snapshot three islands of the $q=8$ chain are deforming the tails of the ensemble; in the second one there are traces of tendrils of the almost completely destroyed $q=7$ chain, while the $q=6$ chain is beginning to be felt; in the last snapshot the $q=6$ chain is almost completely destabilized, but still some tendrils and (on the left side) the remains of a whorl, are visible. (From Ref. [16]) 


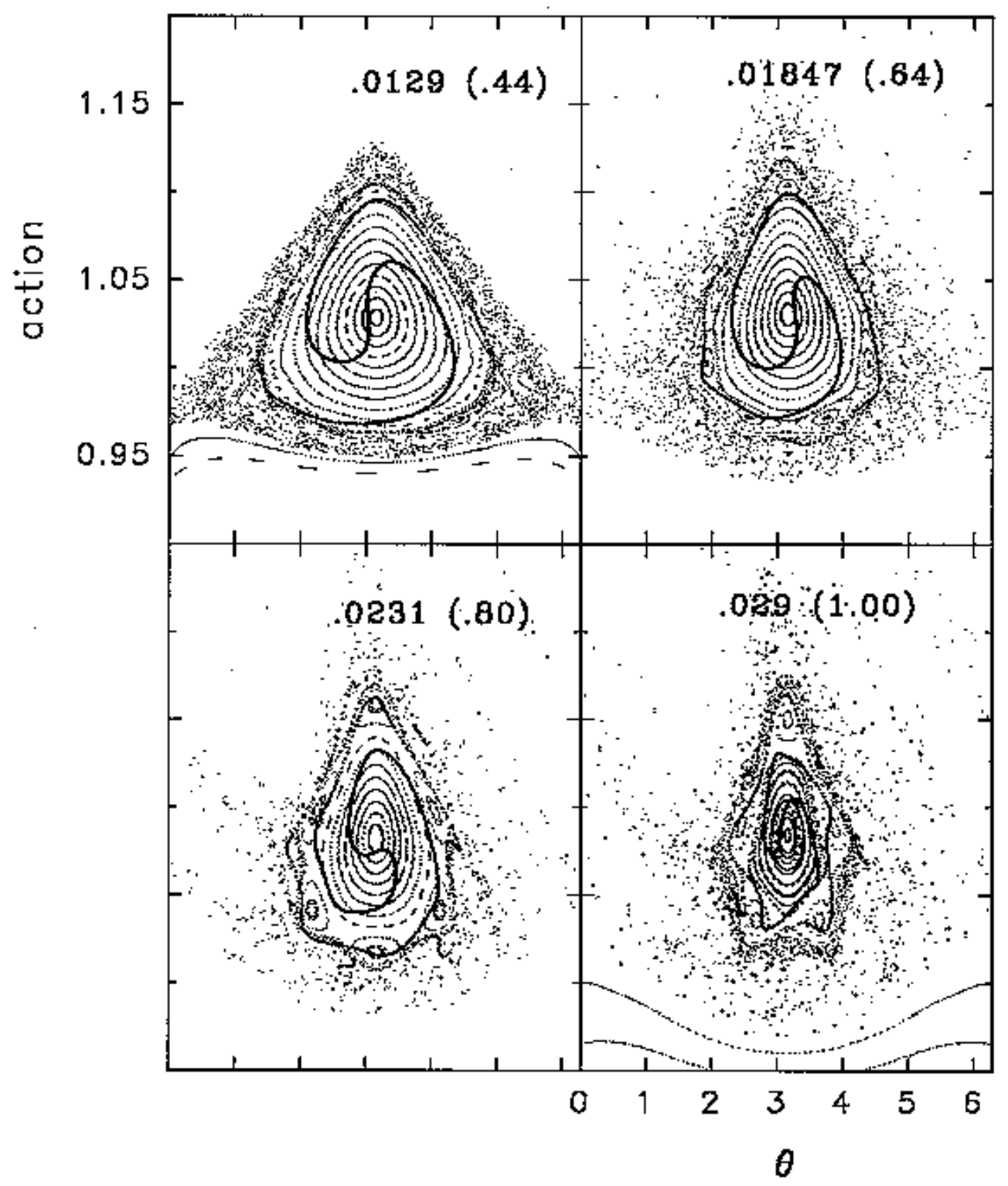

FIG. 8: Comparison of snapshots from Fig. $4\left(F_{0}^{\max }=0.029\right)$ with the surfaces of section calculated at the values of the instantaneous $F_{0}(t) ; F_{0}$ is indicated at the top of each graph, its ratio to $F_{0}^{\text {max }}$ is given in parenthesis. In the second snapshot the $q=7$ chain is beginning to be felt; in the third it is the $q=6$ chain that is deforming the tails of the ensemble; and in the fourth the $q=5$ chain. (From Ref. [16]) 


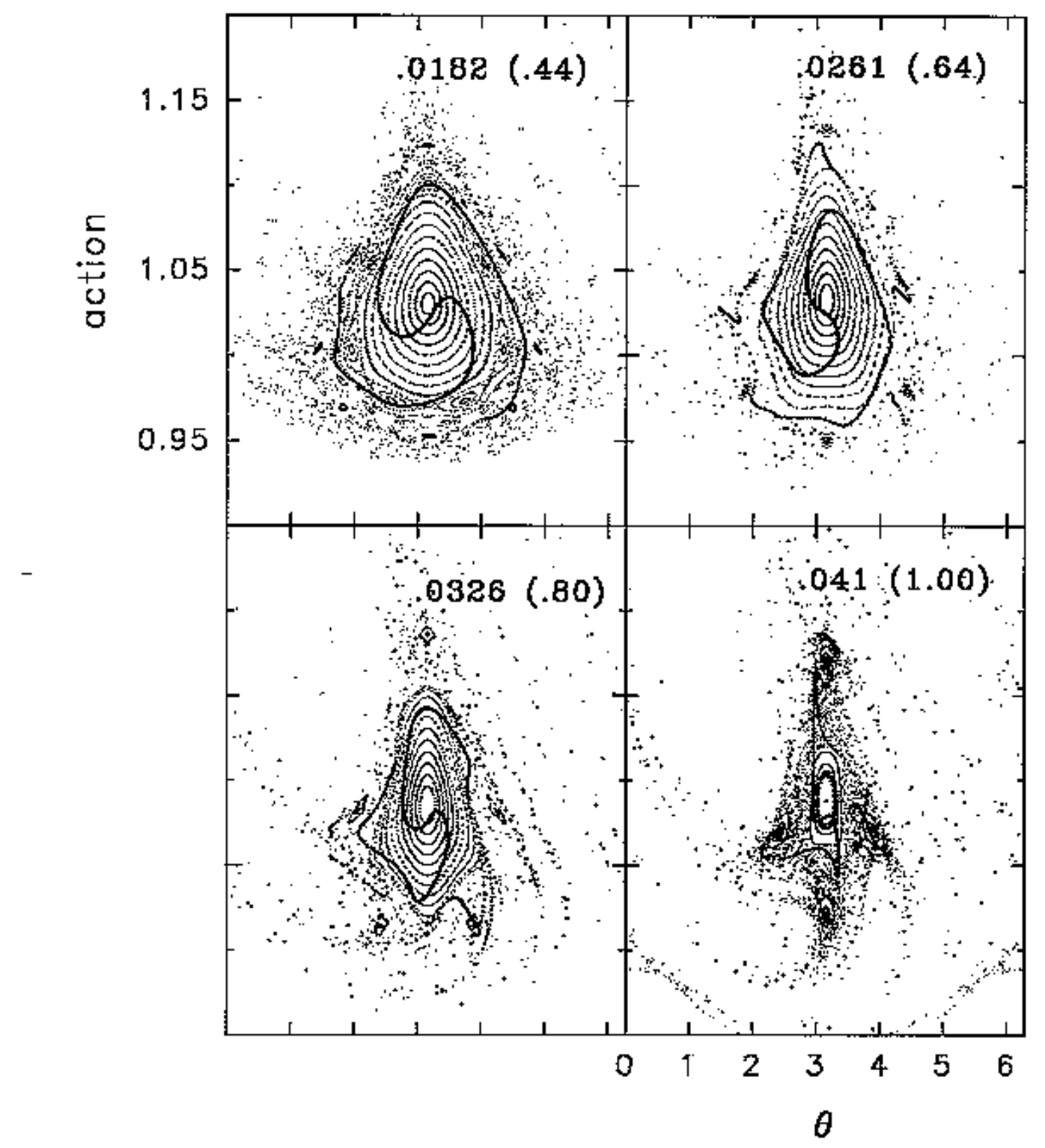

FIG. 9: Comparison of snapshots from Fig. $5\left(F_{0}^{\max }=0.041\right)$ with the surfaces of section calculated at the values of the instantaneous $F_{0}(t) ; F_{0}$ is indicated at the top of each graph, its ratio to $F_{0}^{\max }$ is given in parenthesis. In the first snapshot both the $q=8$ and $q=7$ chains sligtly act on the enemble; but in the second the tendrils of the $q=6$ chain are well developed; in the third the active chain is the $q=5$ one; and in the fourth the $q=4$ one. (From Ref. [16]) 


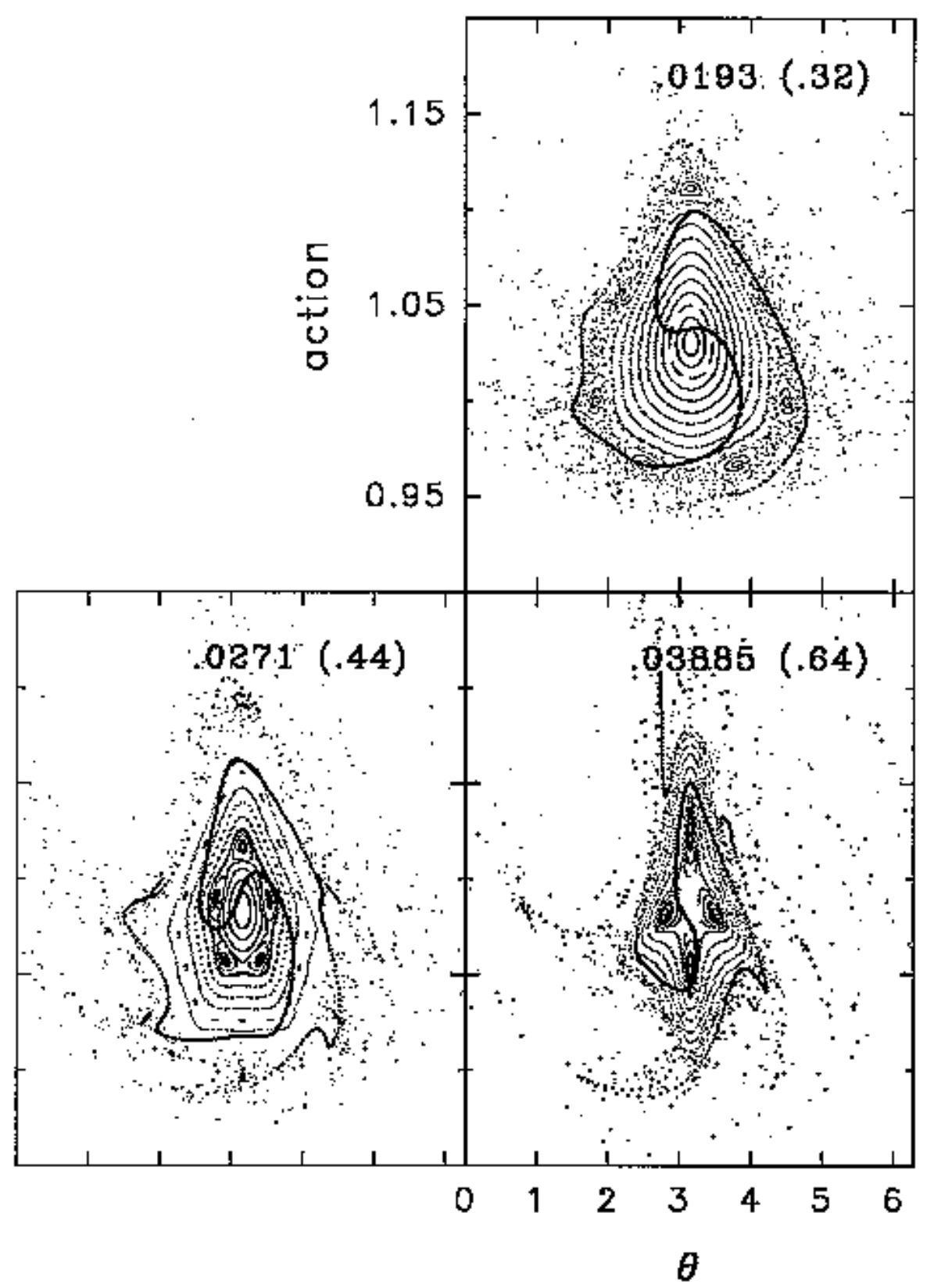




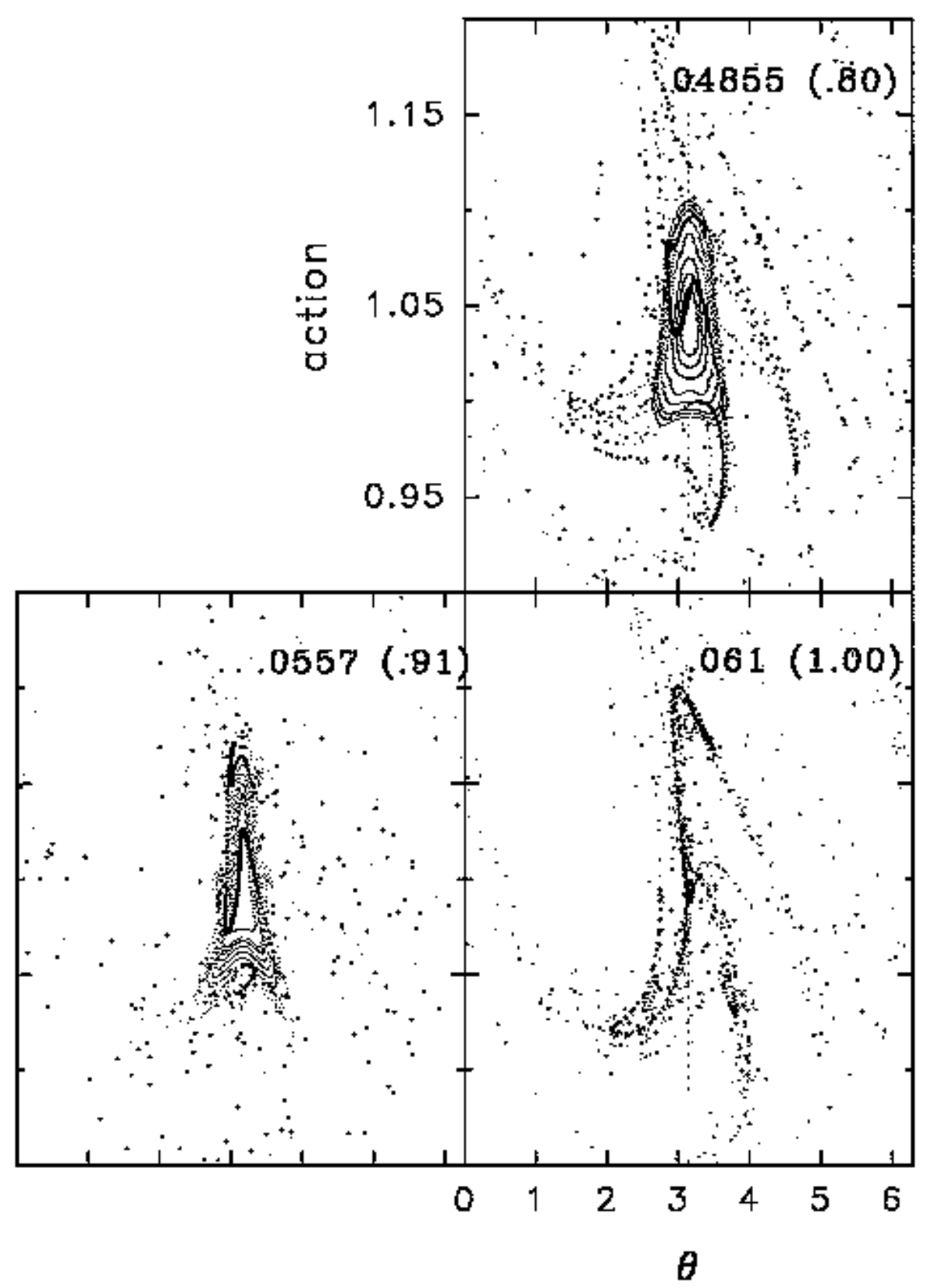

FIG. 10: Comparison of snapshots from Fig. $6\left(F_{0}^{\max }=0.061\right)$ with the surfaces of section calculated at the values of the instantaneous $F_{0}(t) ; F_{0}$ is indicated at the top of each graph, its ratio to $F_{0}^{\max }$ is given in parenthesis. In the first and second snapshots the $q=7$ and $q=6$ chains respectively are the cause of the deformations of the tails of the primary island whorl; in the third snapshot the $q=4$ chain begins to act but the tendrils of the $q=5$ one are still visible; so in the fourth the tendrils of the $q=4$ chain; the fifth and sixth snapshots see the creation and development of the $q=3$ tendrils (no stability islands are present for $q=3$ ). (From Ref. [16]) 

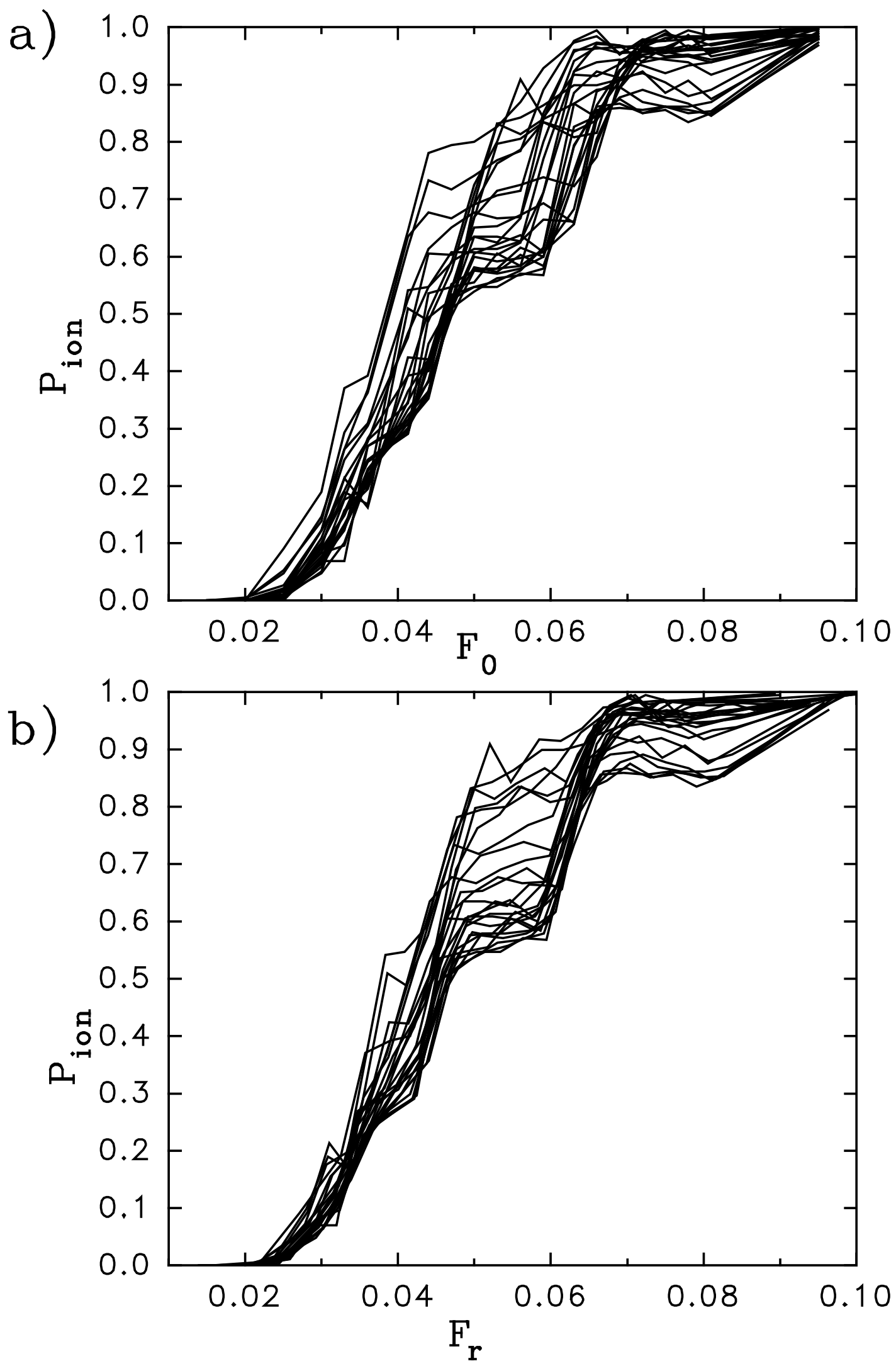

FIG. 11: a) The classical probability for microwave ionization of $n_{0}=69$ atoms plotted as a function of the rescaled peak microwave field strength $F_{0}^{\text {max }}$. There is no alignment of the ionization steps of the 25 curves shown (at values of $\omega_{0}^{\prime}$ equispatiated between $\omega_{0}^{\prime}=0.9325$ and $\left.\omega_{0}^{\prime}=1.0666\right)$. b) The same but plotted as a function of peak microwave field strength rescaled at the resonance $F_{r}^{\max }$. With this choice of the horizontal scale the ionization steps are aligned. (From Ref. [16]) 

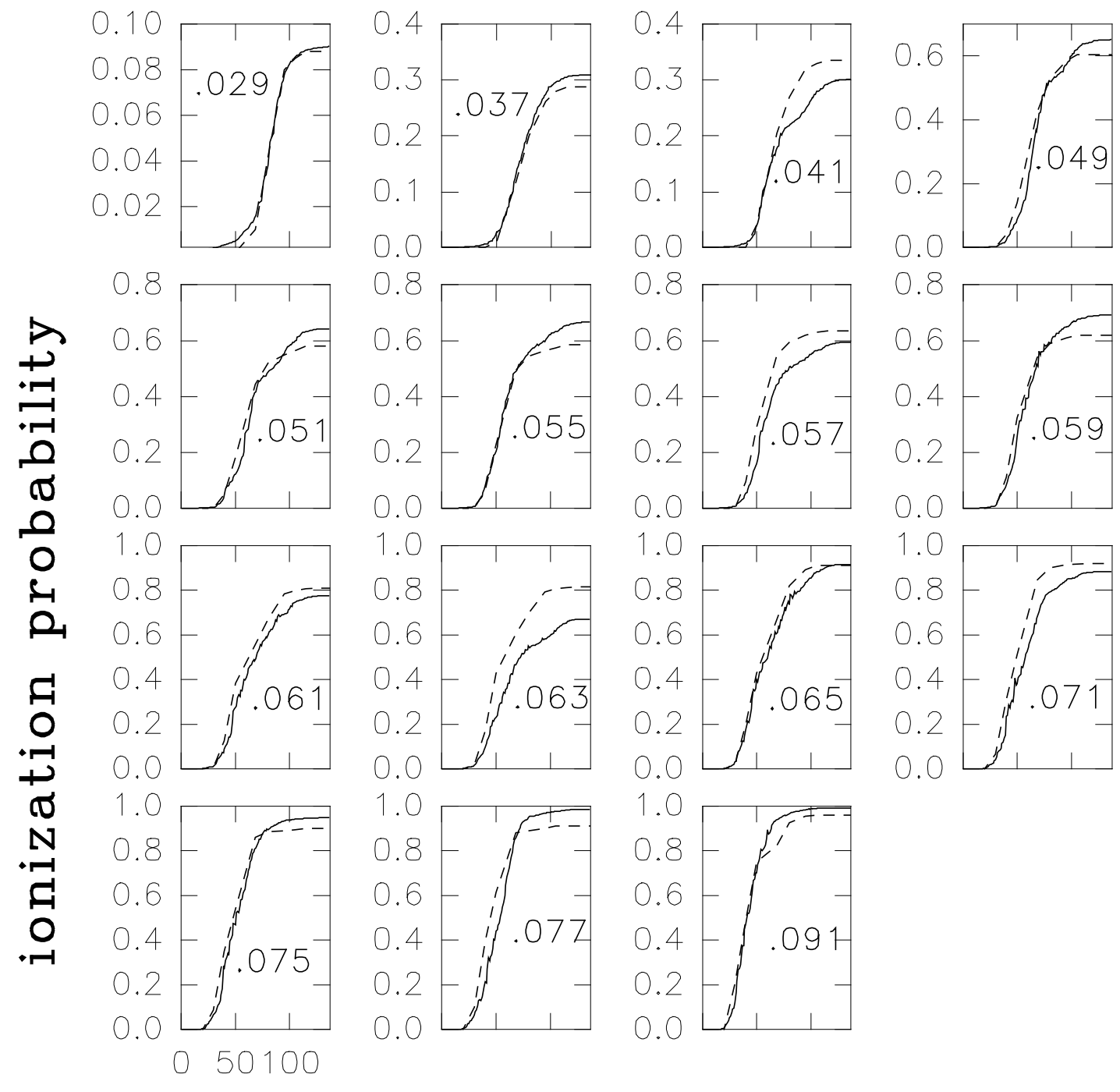

0.0

0.0
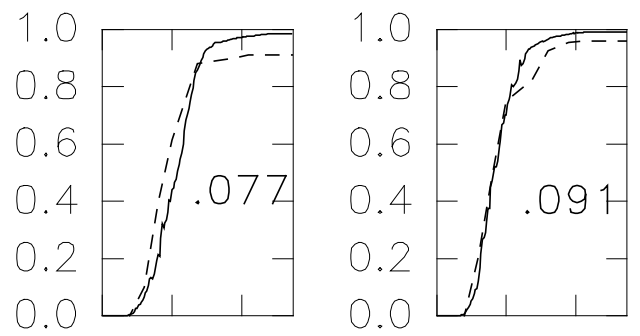

0.0

\section{time}

FIG. 12: Comparison of quantum (full line) and classical (dashed line) time evolutions for various values of $F_{0}^{\max }$ (indicated next to each plot). $F_{0}^{S}=0.035266$ and $\omega_{0}^{\prime}=0.9771$. (From Ref. [16]) 


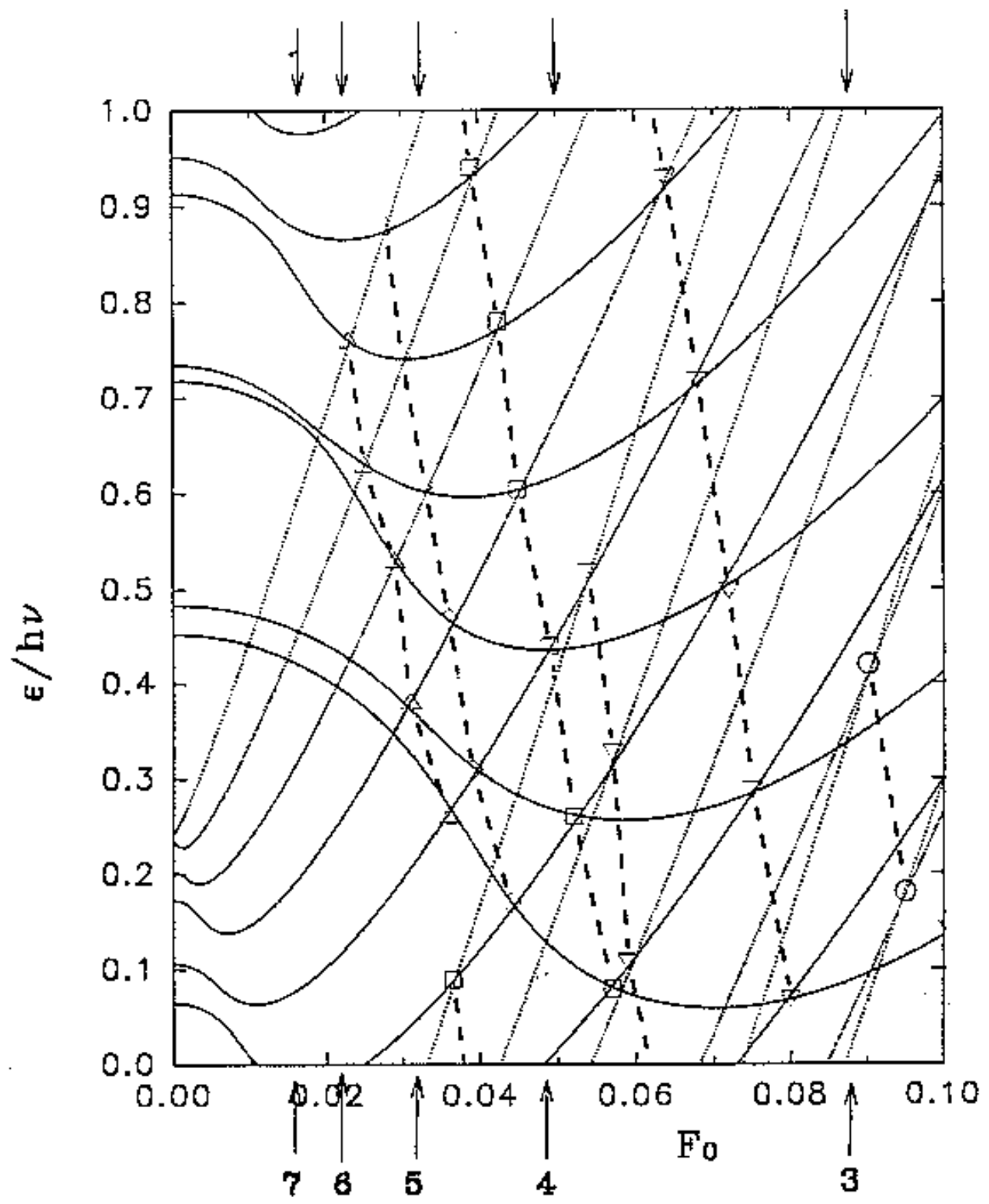

FIG. 13: Pendulum approximation quasienergy curves for the case of Fig. 1. The groups of crossings related to the most visible classical secondary island chains are indicated by the same symbols as in Fig. 1. Here the arrows mark the classical approximate bifurcation fields eq (B7), labeled by $q$. (From Ref. [16]) 


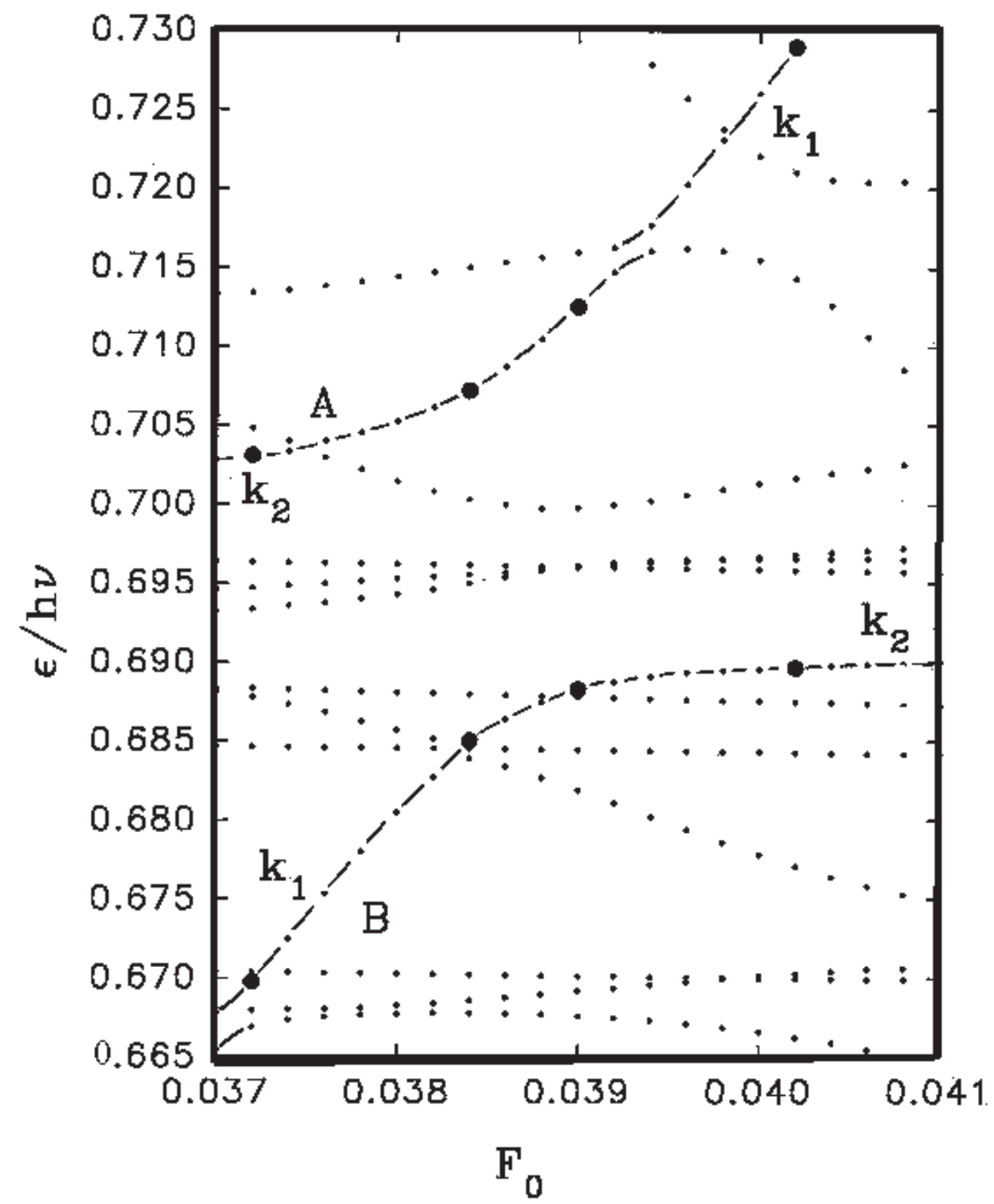



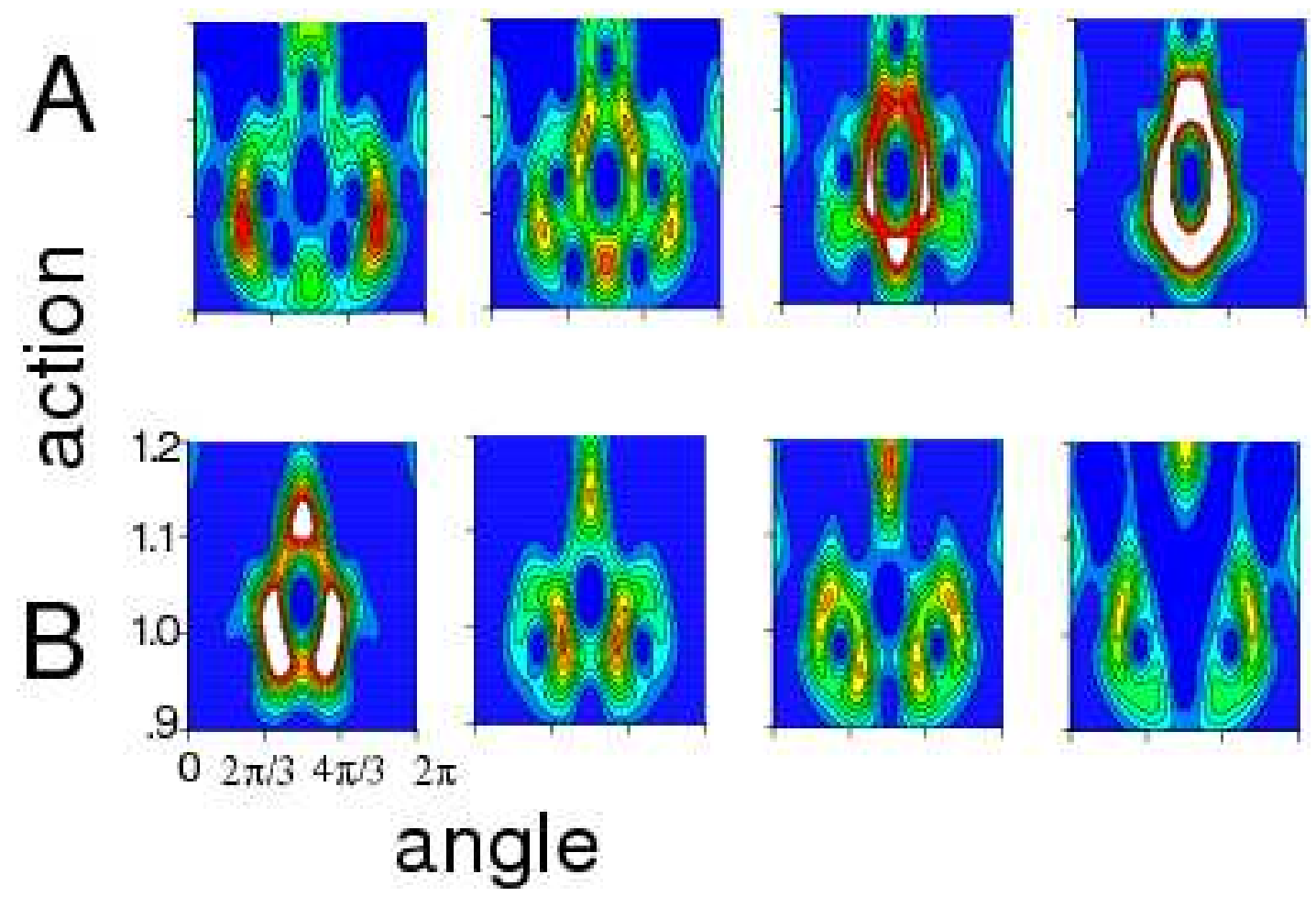

\section{angle}

FIG. 14: Detail of Fig. 1 showing one of the avoided crossings marked in Fig. 1 and the Husimi functions of the (eigen-)states undergoing this same avoided crossing: it is a wide crossing where no other interacting level intervenes: the third $q=5$ crossing $\left(k_{1}=2, k_{2}=7\right)$. The (adiabatic) states are labeled by letters and numbers indicate the (diabatic) resonance quantization $k$. Bigger dots mark on the quasienergy curves the points corresponding to the Husimi functions displayed. Lighter shades of gray between the level curves mean higher values of the Husimi functions. The levels of the curves is the same for all plots; the highest peaks in some of them are, as a result, out of range and appear as white areas. 


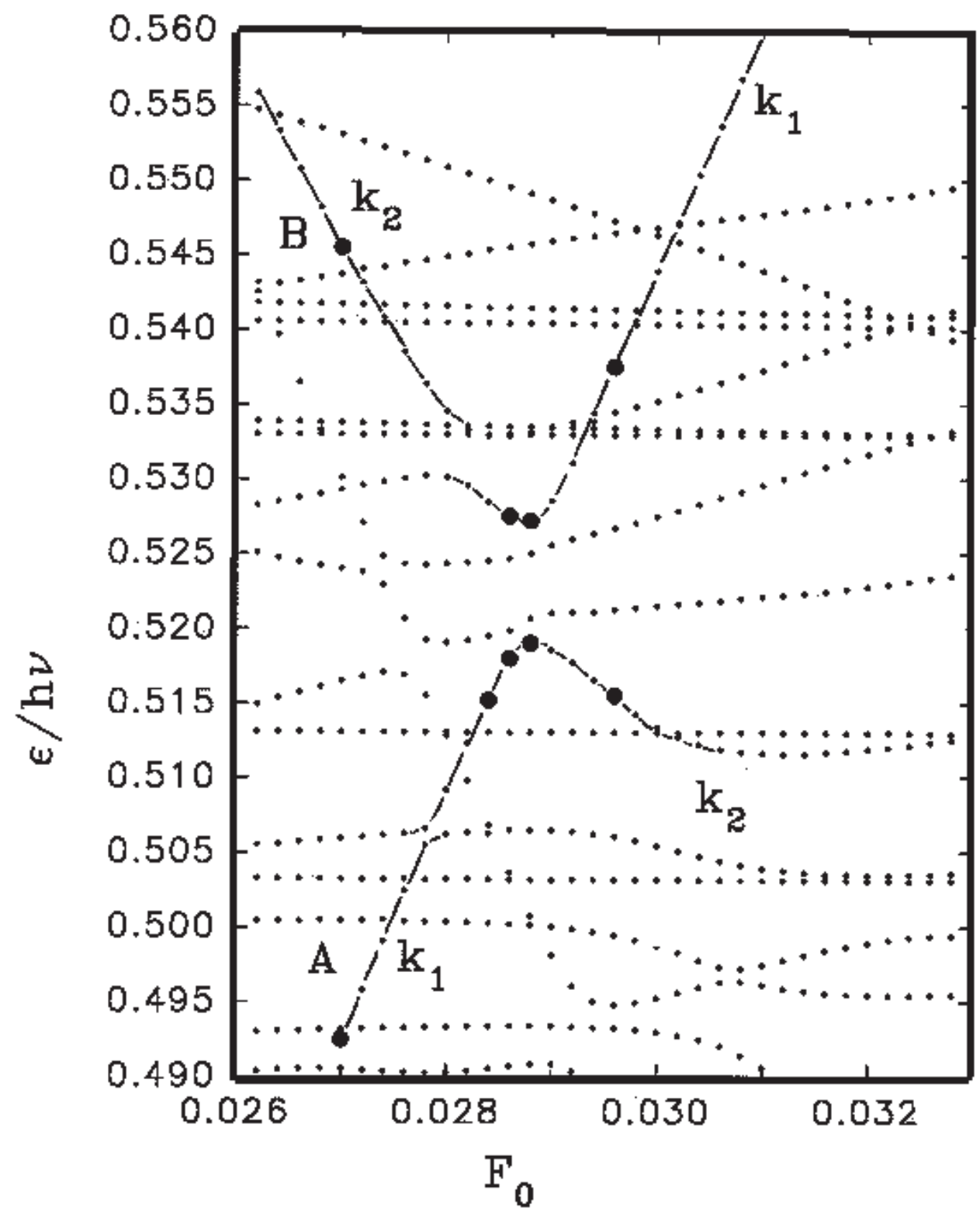




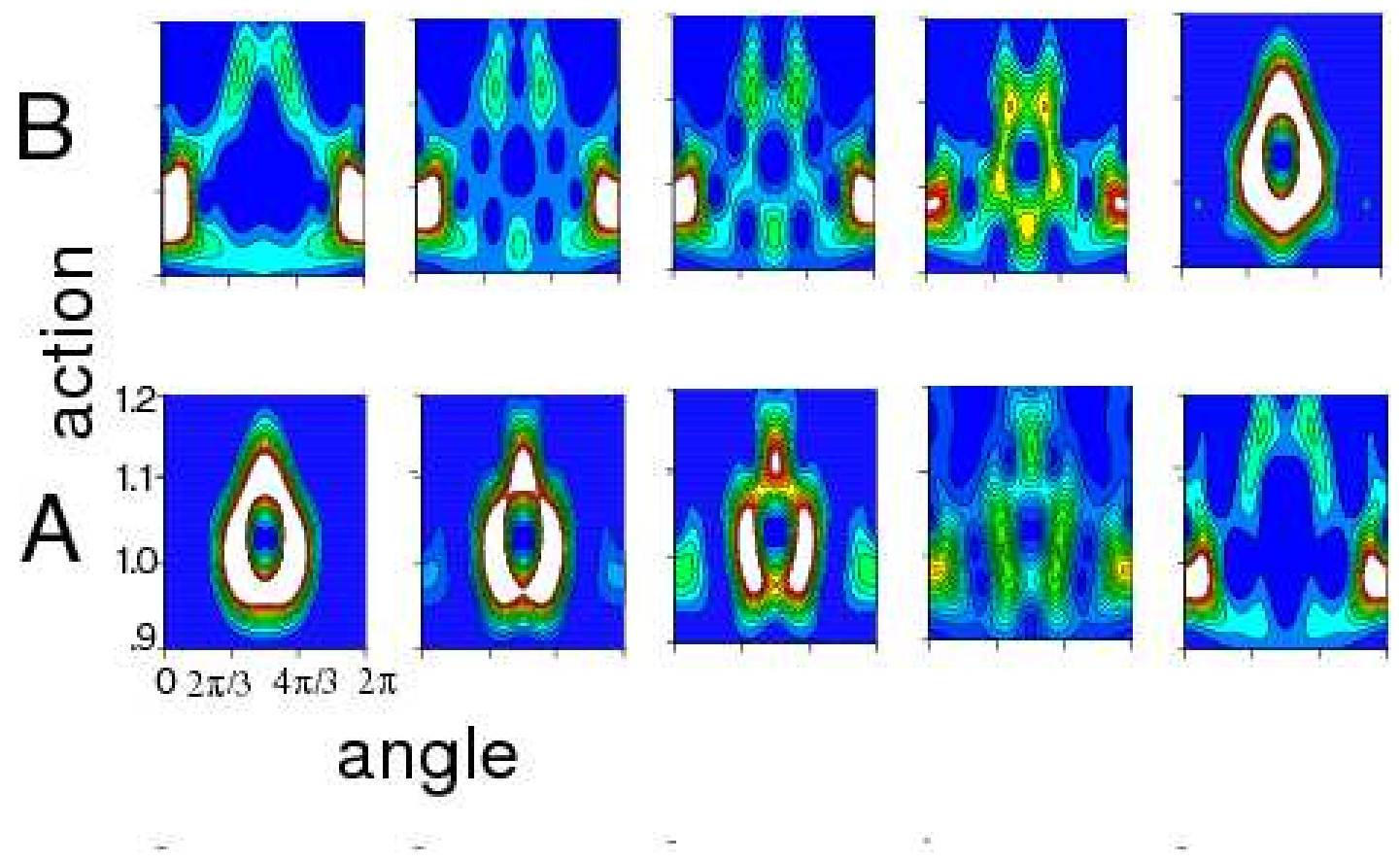

FIG. 15: Detail of Fig. 1 showing one of the avoided crossings marked in Fig. 1 and the Husimi functions of the (eigen-)states undergoing this same avoided crossing: it is a narrow crossing where no other level intervenes: the third $q=7$ crossing $\left(k_{1}=2\right.$, $k_{2}=9$ ). The (adiabatic) states are labeled by letters and numbers indicate the (diabatic) resonance quantization $k$. Bigger dots mark on the quasienergy curves the points corresponding to the Husimi functions displayed. Lighter shades of gray between the level curves mean higher values of the Husimi functions. The levels of the curves is the same for all plots; the highest peaks in some of them are, as a result, out of range and appear as white areas. 


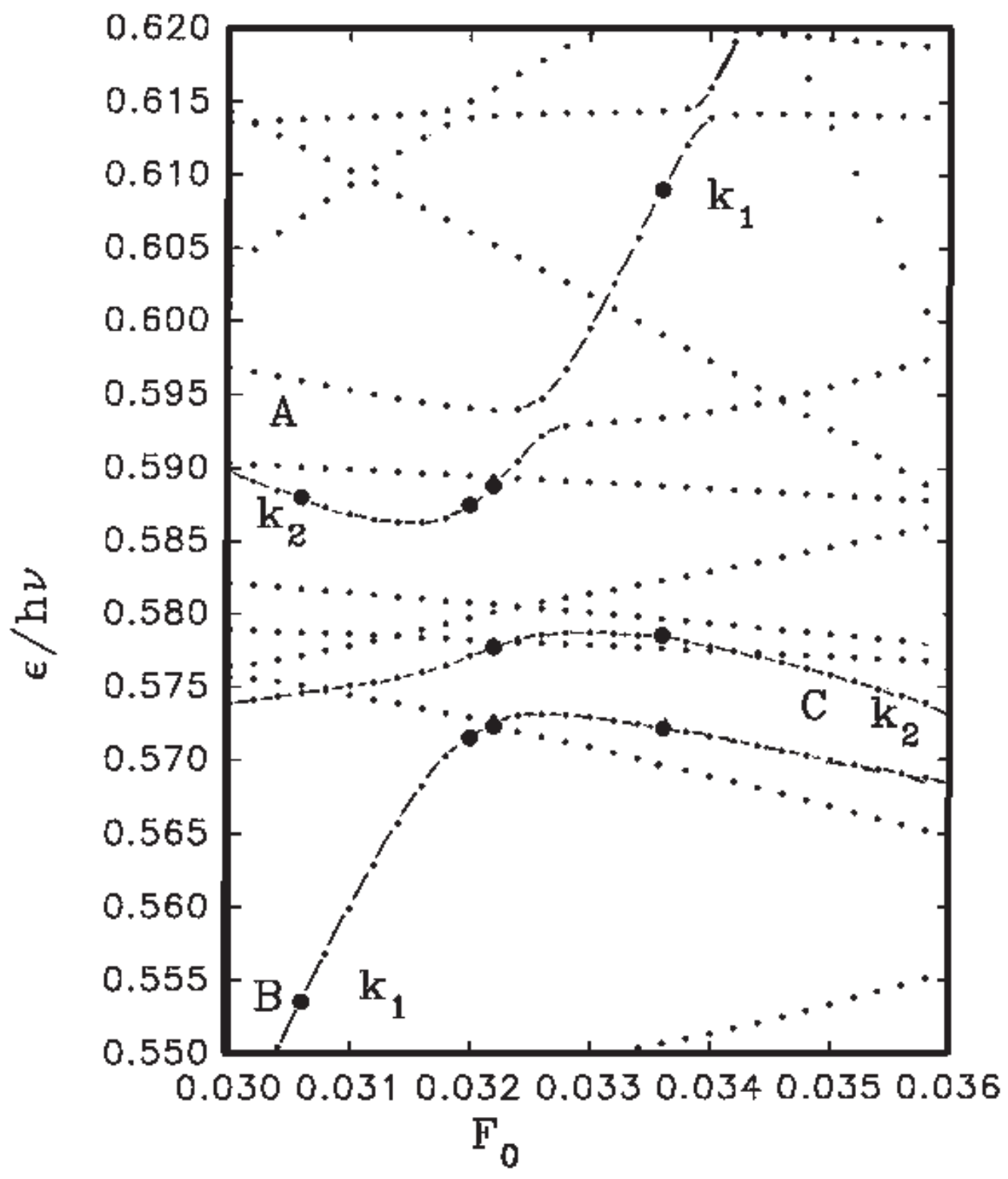



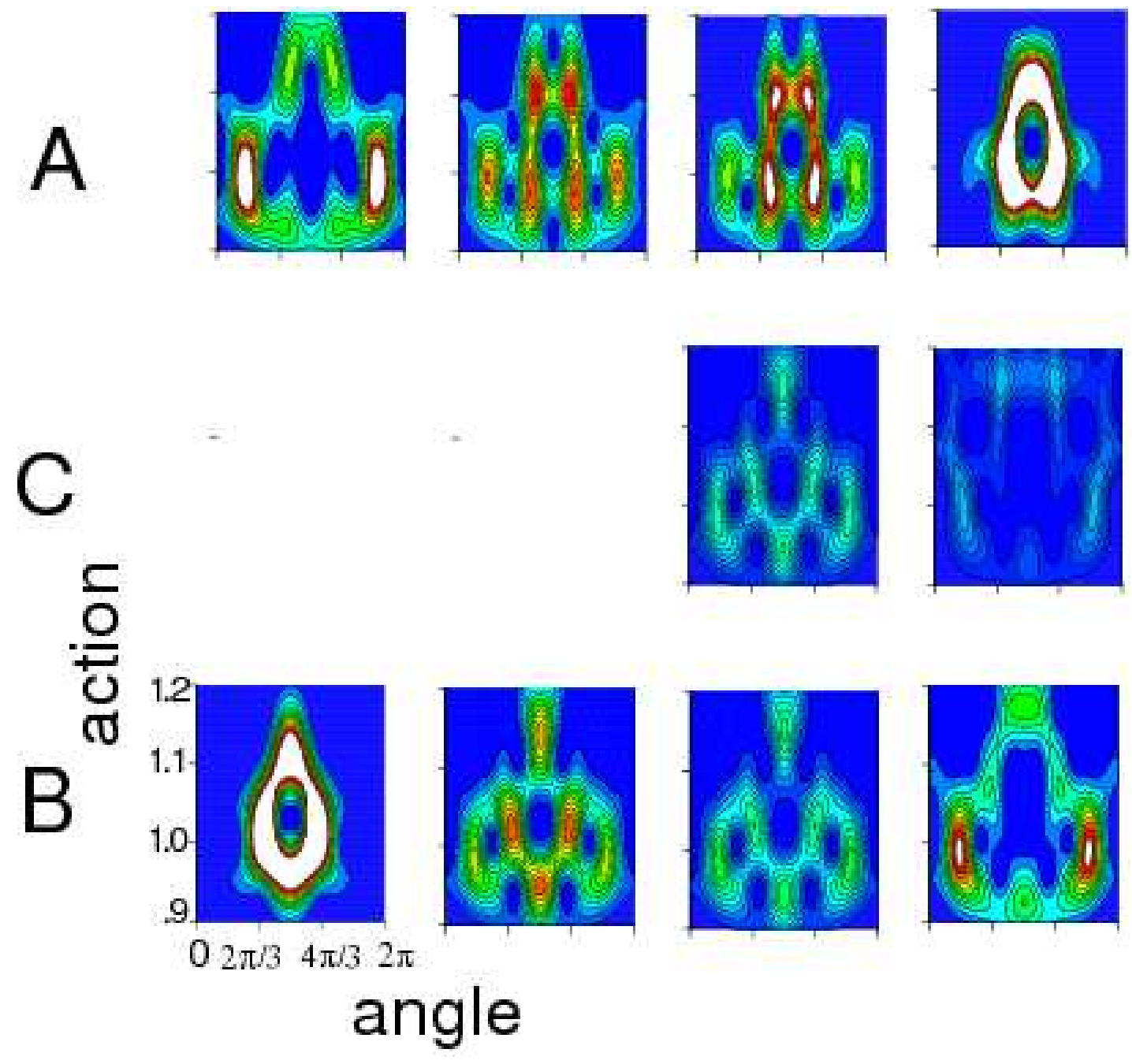

FIG. 16: Detail of Fig. 1 showing one of the avoided crossings marked in Fig. 1 and the Husimi functions of the (eigen-)states undergoing this same avoided crossing: it is a wide crossing where the lower "adiabatic" state is made up of two states (B and $\mathrm{C}$ ): the third $q=6$ crossing $\left(k_{1}=2, k_{2}=8\right)$. The (adiabatic) states are labeled by letters and numbers indicate the (diabatic) resonance quantization $k$. Bigger dots mark on the quasienergy curves the points corresponding to the Husimi functions displayed. Lighter shades of gray between the level curves mean higher values of the Husimi functions. The levels of the curves is the same for all plots; the highest peaks in some of them are, as a result, out of range and appear as white areas. 


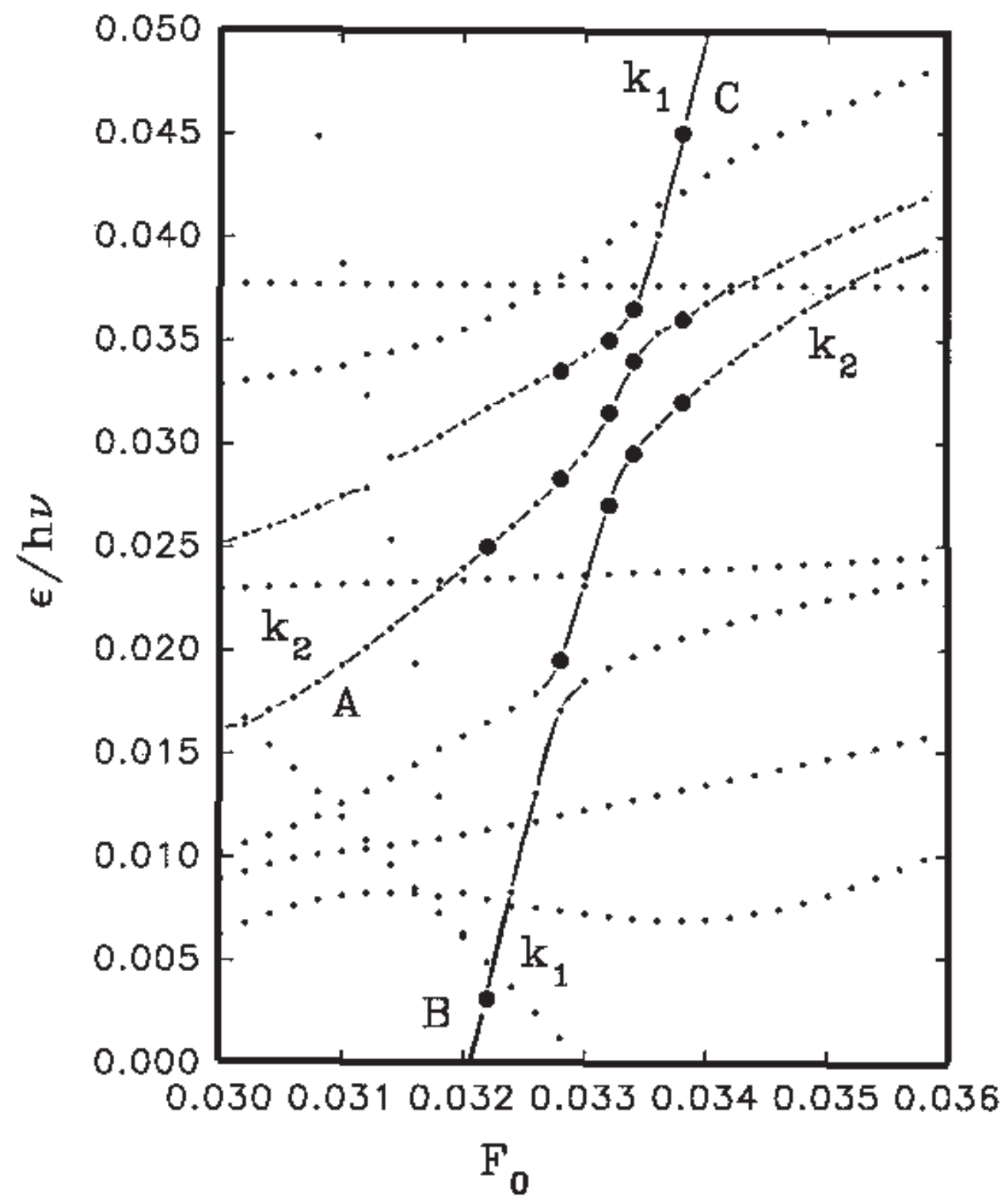



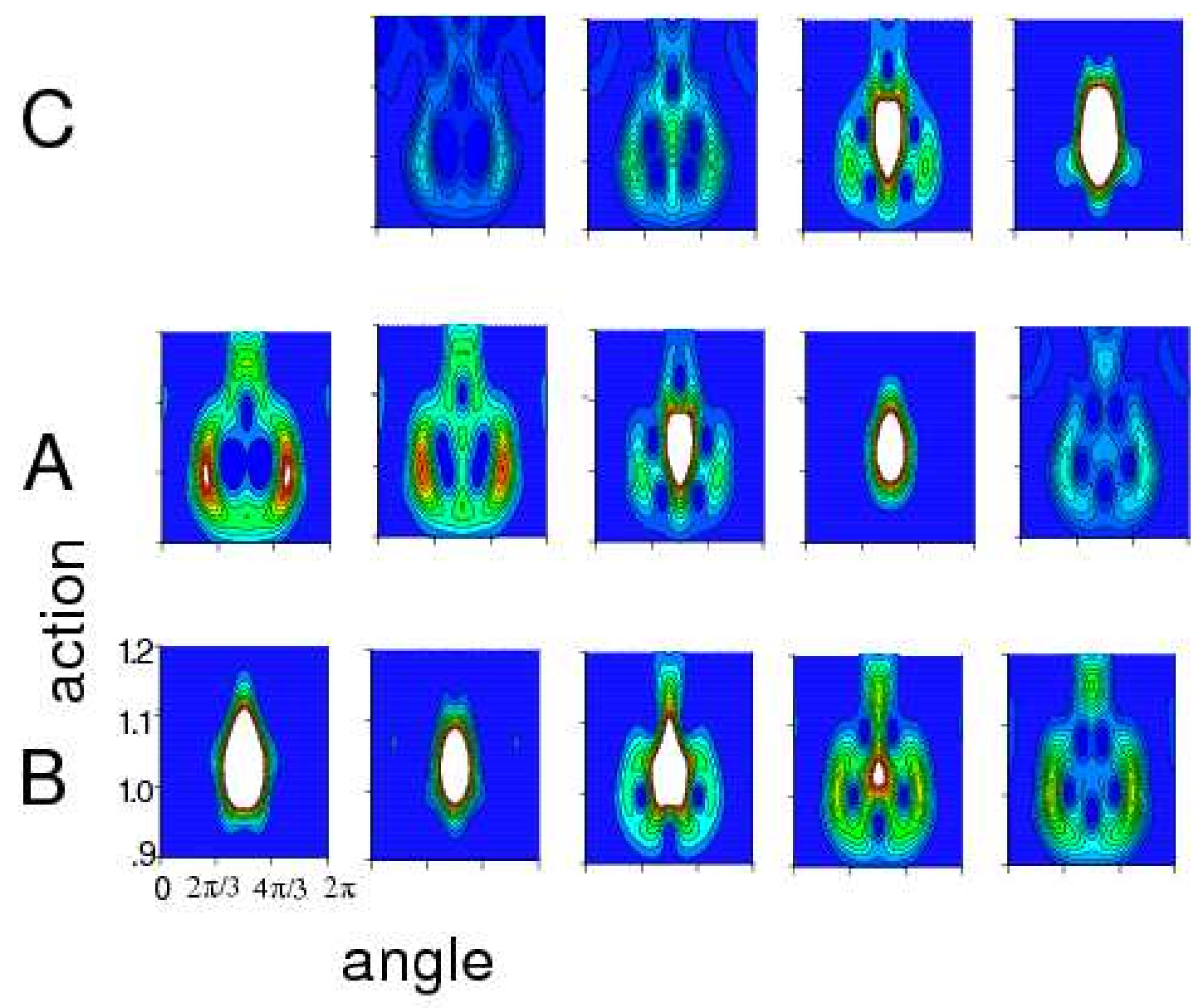

FIG. 17: Detail of Fig. 1 showing one of the avoided crossings marked in Fig. 1 and the Husimi functions of the (eigen-)states undergoing this same avoided crossing: it is a wide crossing where the upper "adiabatic" state is made up of two states (A and $\mathrm{C}$ ): the first $q=5$ crossing $\left(k_{1}=0, k_{2}=5\right)$. The (adiabatic) states are labeled by letters and numbers indicate the (diabatic) resonance quantization $k$. Bigger dots mark on the quasienergy curves the points corresponding to the Husimi functions displayed. Lighter shades of gray between the level curves mean higher values of the Husimi functions. The levels of the curves is the same for all plots; the highest peaks in some of them are, as a result, out of range and appear as white areas. 


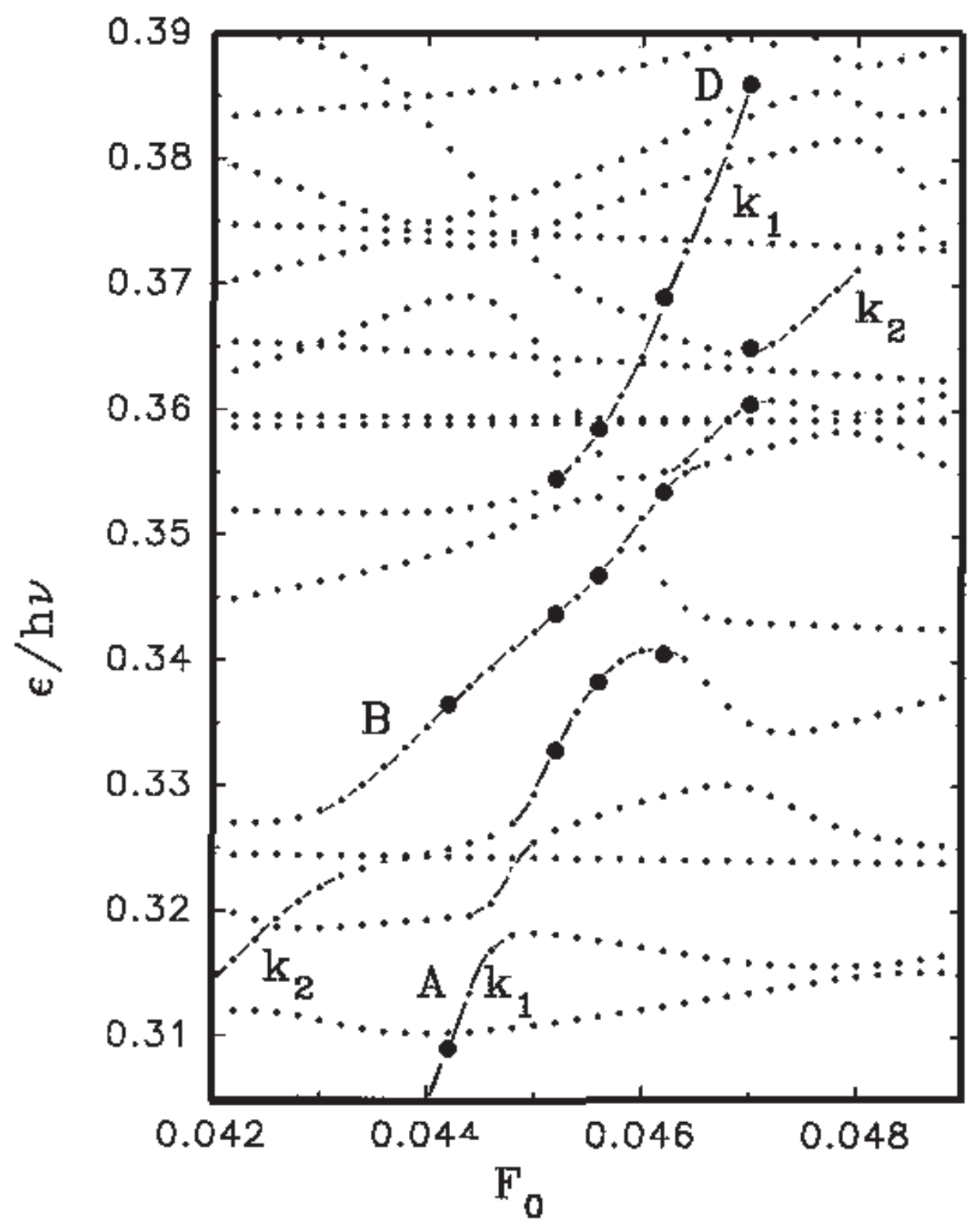



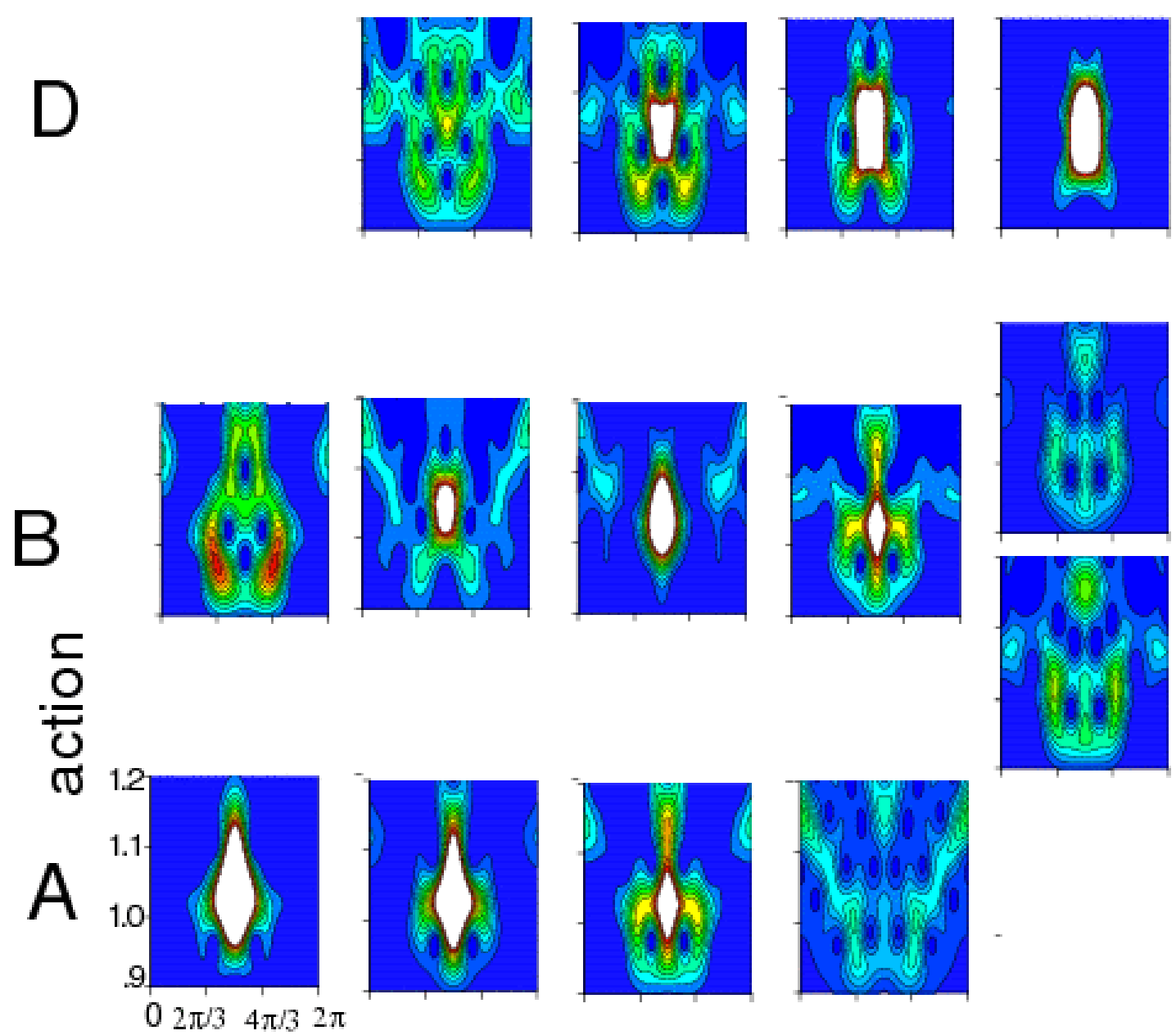

\section{angle}

FIG. 18: Detail of Fig. 1 showing one of the avoided crossings marked in Fig. 1 and the Husimi functions of the (eigen-)states undergoing this same avoided crossing: it is a wwide crossing where a crossing state (B) maintains the same (diabatic) resonance quantum number $k_{2}=4$ : the first $q=4$ crossing $\left(k_{1}=0, k_{2}=4\right)$. The (adiabatic) states are labeled by letters and numbers indicate the (diabatic) resonance quantization $k$. Bigger dots mark on the quasienergy curves the points corresponding to the Husimi functions displayed. Lighter shades of gray between the level curves mean higher values of the Husimi functions. The levels of the curves is the same for all plots; the highest peaks in some of them are, as a result, out of range and appear as white areas. 


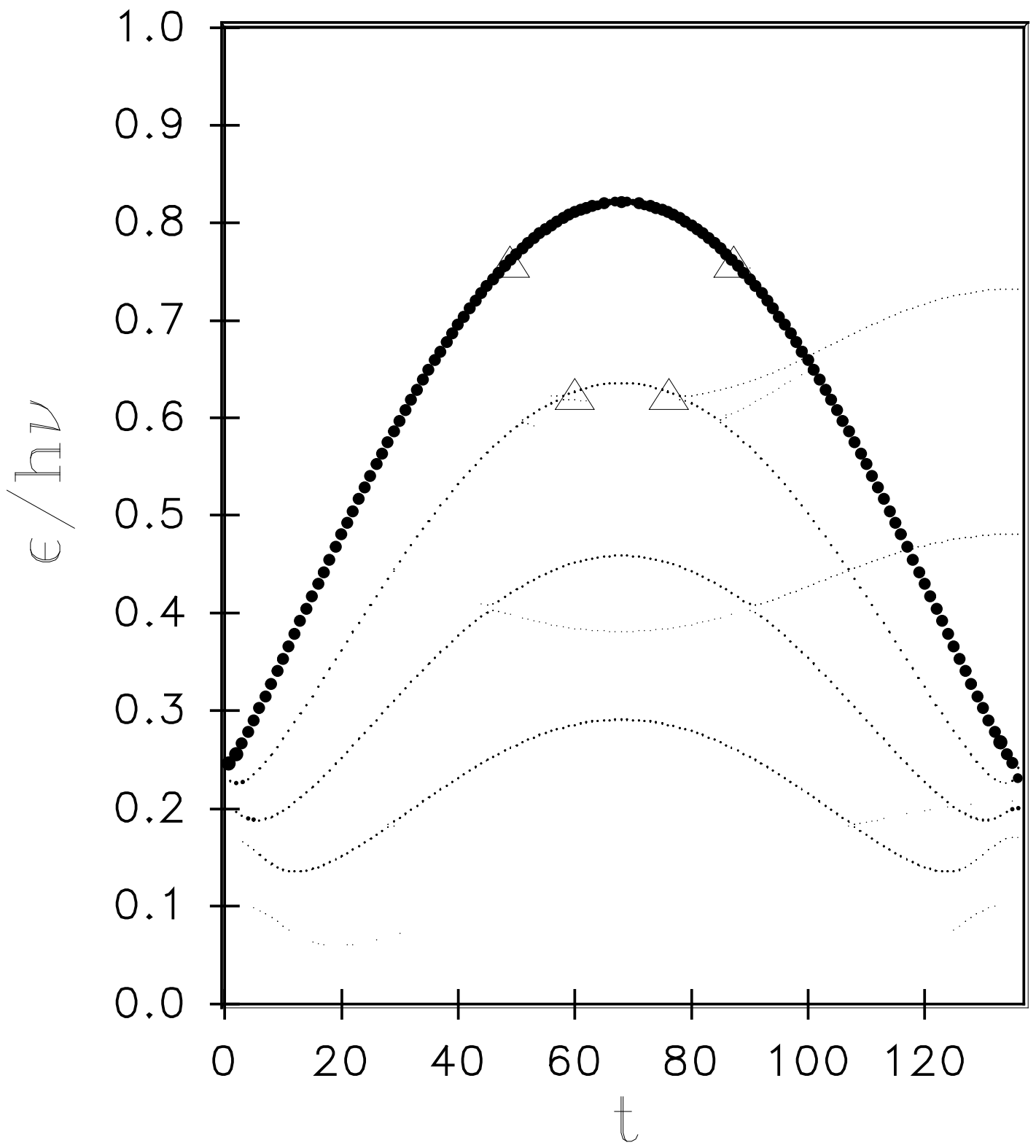

FIG. 19: The quasienergies of the states most significantly populated at every period of the microwave pulse for the case of Fig. $3\left(F_{0}^{\max }=0.025\right)$. The (linear) size of each dot is proportional to the population on the corresponding level at that point, the minimum size corresponding to a $0.5 \%$ population. The same symbols as in Fig. 1 mark the avoided crossings related to classical secondary resonance island chains. 


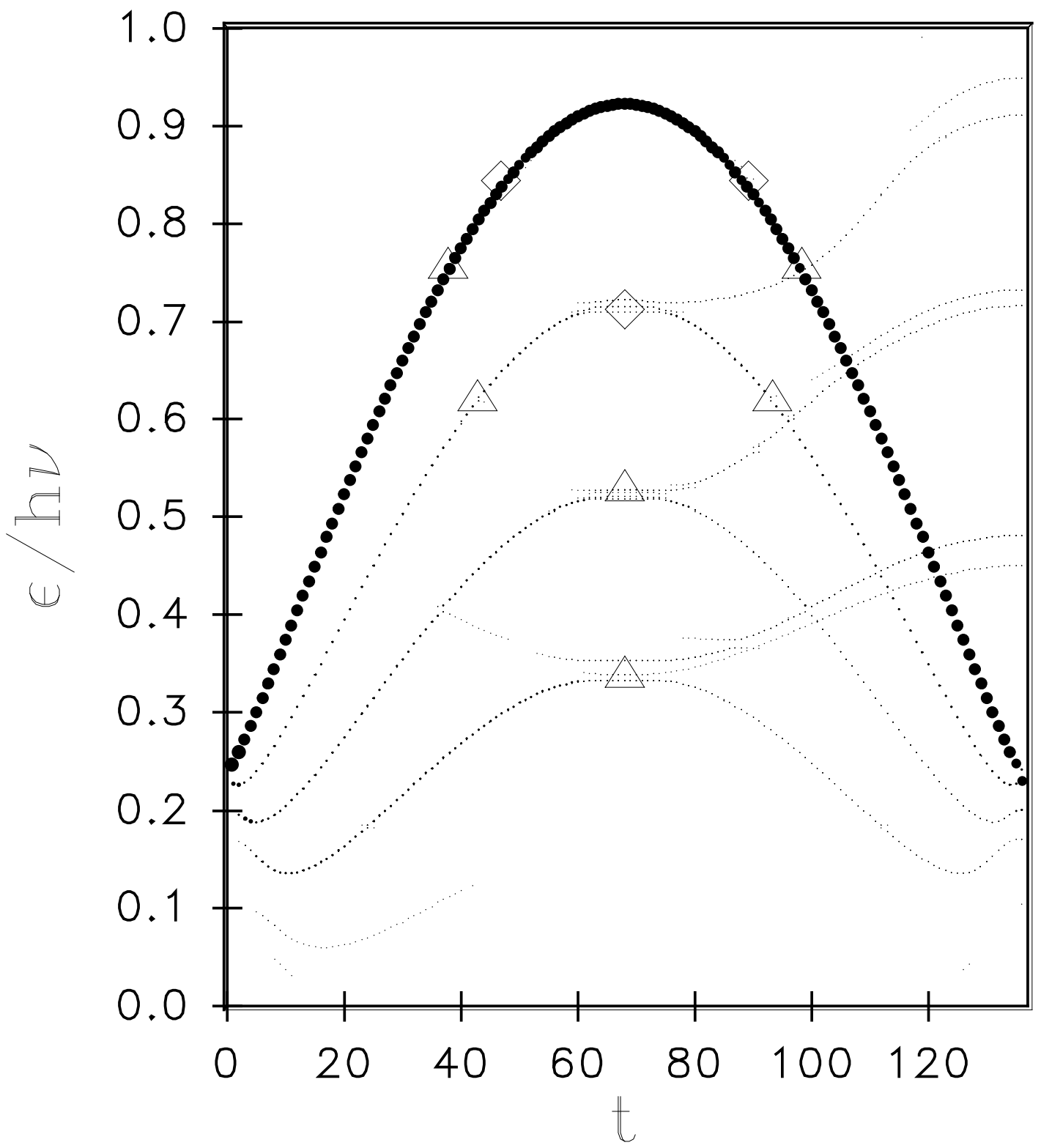

FIG. 20: The quasienergies of the states most significantly populated at every period of the microwave pulse for the case of Fig. $4\left(F_{0}^{\max }=0.029\right)$. The (linear) size of each dot is proportional to the population on the corresponding level at that point, the minimum size corresponding to a $0.5 \%$ population. The same symbols as in Fig. 1 mark the avoided crossings related to classical secondary resonance island chains. 


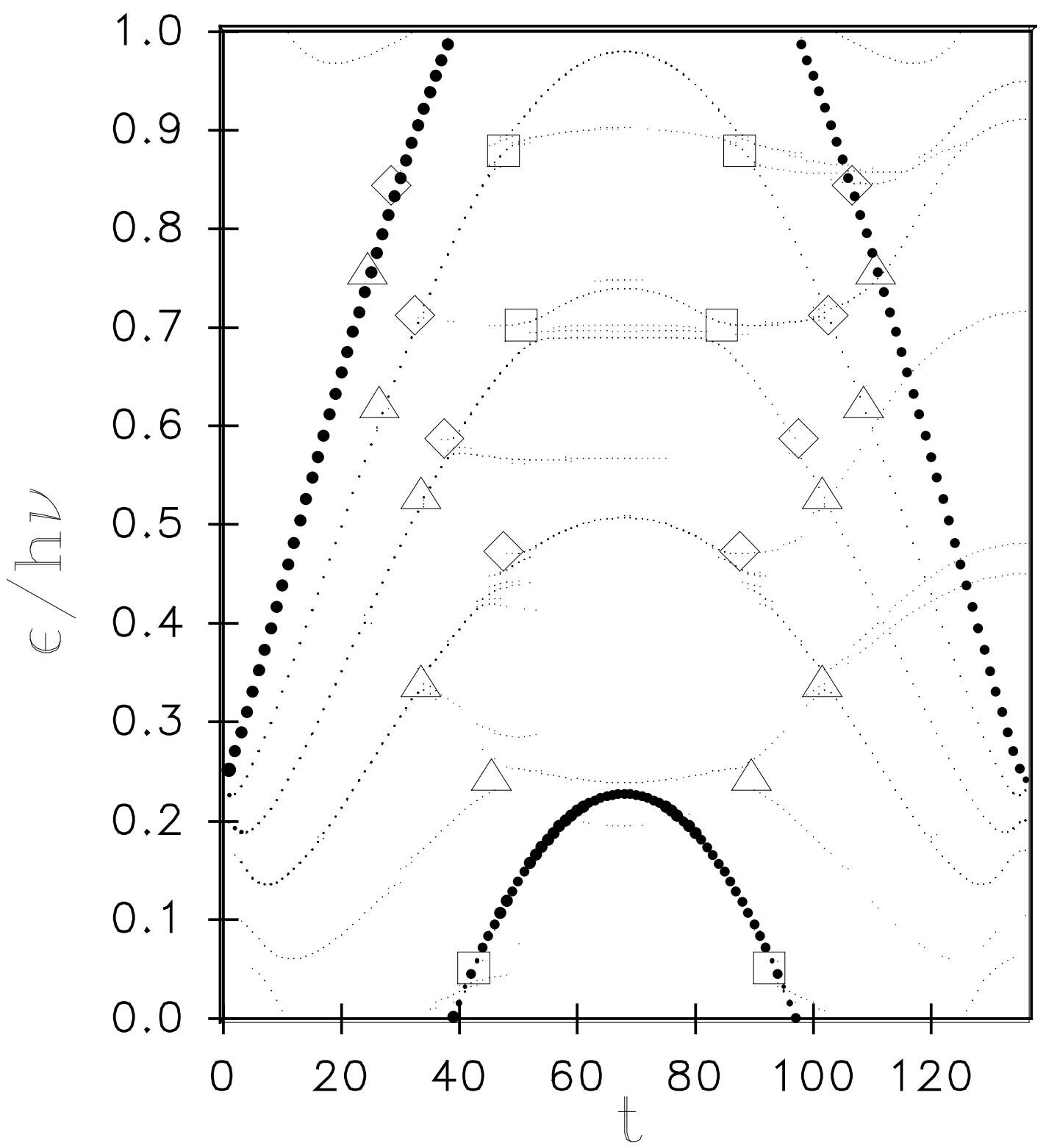

FIG. 21: The quasienergies of the states most significantly populated at every period of the microwave pulse for the case of Fig. $5\left(F_{0}^{\max }=0.041\right)$. The (linear) size of each dot is proportional to the population on the corresponding level at that point, the minimum size corresponding to a $0.5 \%$ population. The same symbols as in Fig. 1 mark the avoided crossings related to classical secondary resonance island chains. 


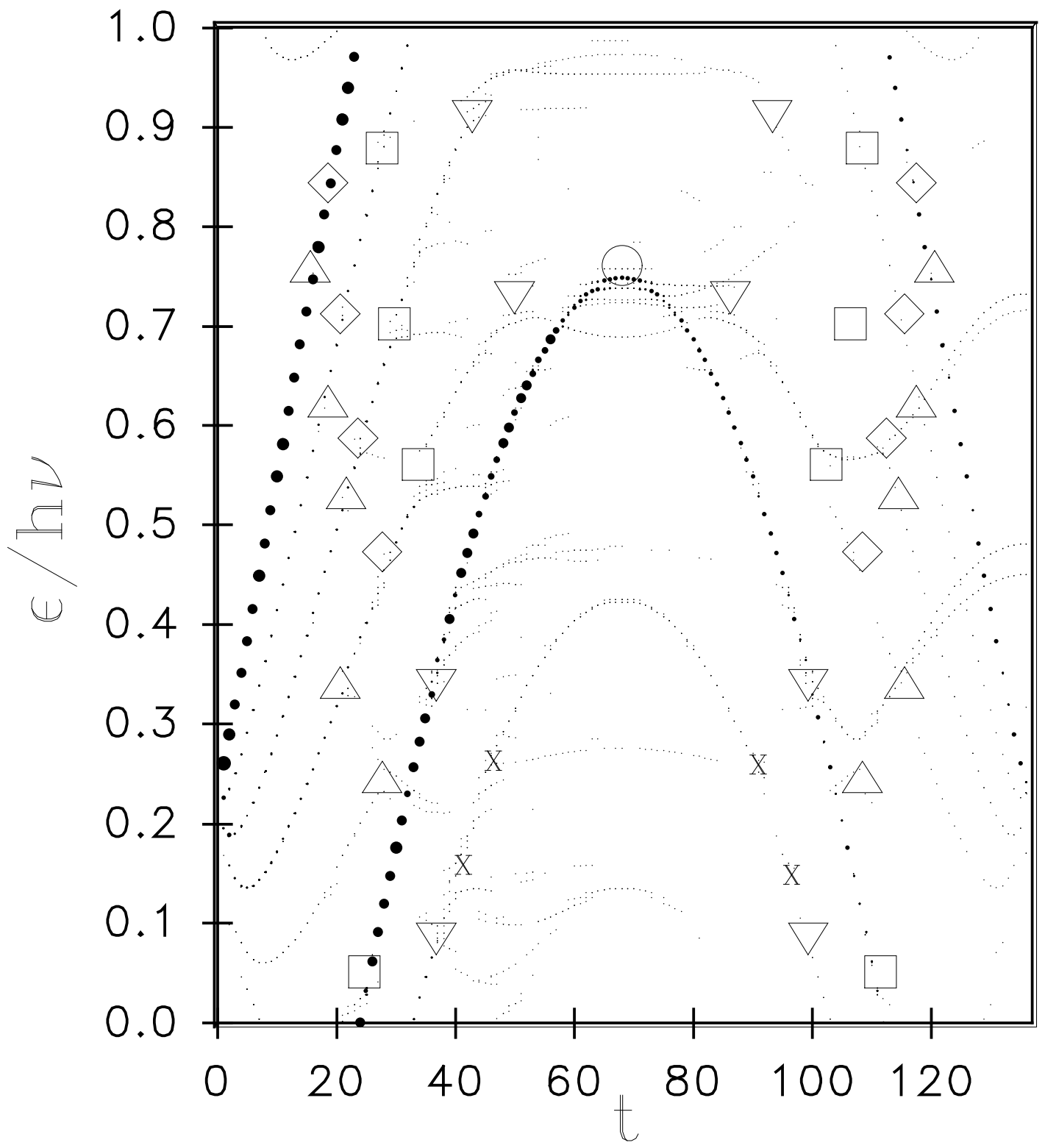

FIG. 22: The quasienergies of the states most significantly populated at every period of the microwave pulse for the case of Fig. $6\left(F_{0}^{\max }=0.061\right)$. The (linear) size of each dot is proportional to the population on the corresponding level at that point, the minimum size corresponding to a $0.5 \%$ population. The same symbols as in Fig. 1 mark the avoided crossings related to classical secondary resonance island chains. The crosses mark crossings with $p=2$ and -from top to bottom- $q=9,9$ and 10 . 


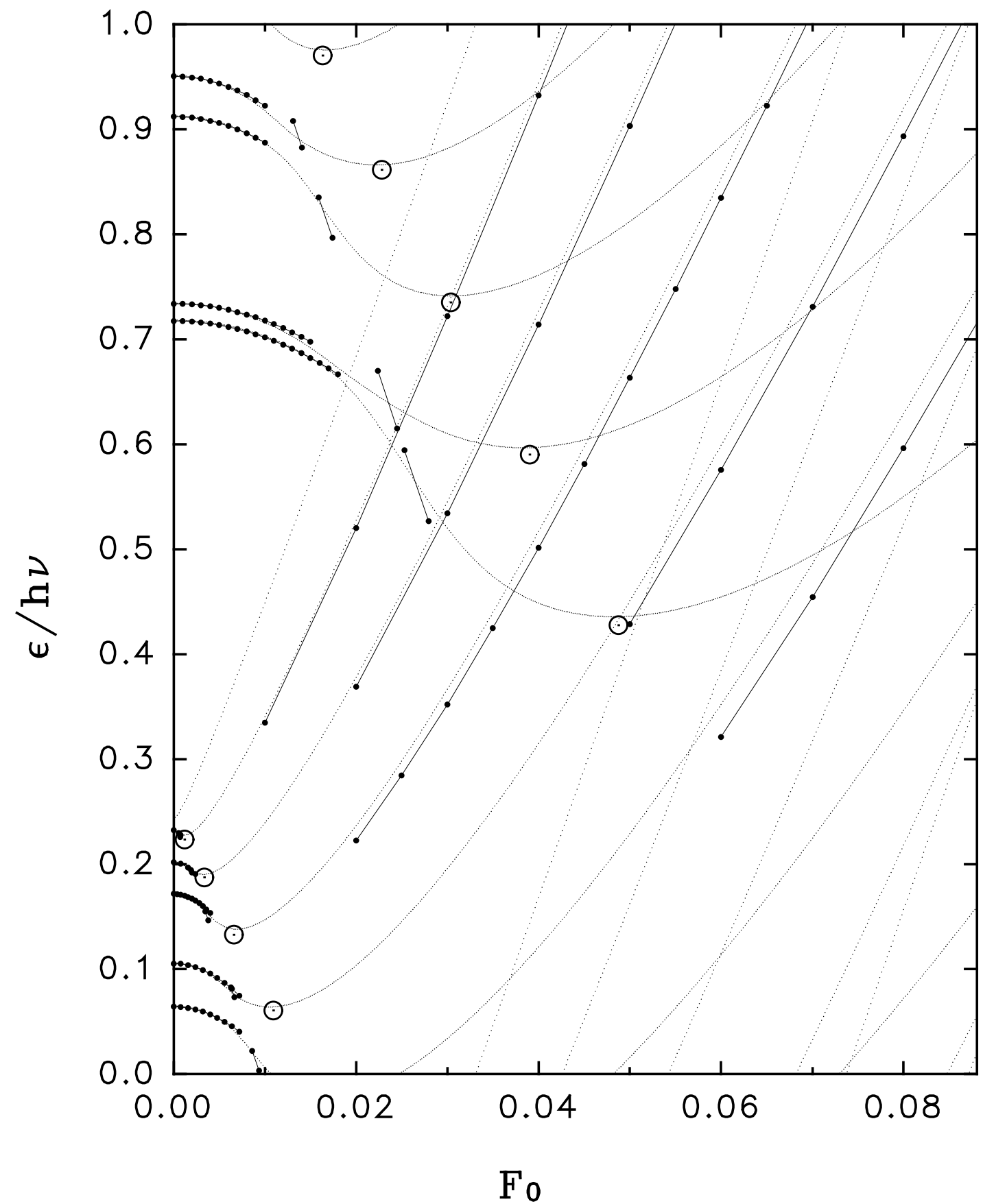

FIG. 23: Comparison of the $k=0-9$ curves obtained from eq. (B9) using the off diagonal matrix elements (B10) (small dots) with the classical prediction (big dots) W.K.B. quantized according to the free atom quantum number $n$ outside the separatrix and according to the resonance quantum number $k$ inside. Both quantizations are shown at the separatrix itself, connected by a segment of straight line. (From Ref. [16]) 